\title{
The intersection of recurrence sequences
}

\author{
by \\ Hans Peter Schlickewei (Ulm) and \\ Wolfgang M. Schmidt (Boulder, Colo.)
}

1. Introduction. Let $\left\{u_{m}\right\}_{m \in \mathbb{Z}}$ be a linear recurrence sequence, i.e., a sequence satisfying a relation

$$
u_{m+k}=\nu_{k-1} u_{m+k-1}+\ldots+\nu_{1} u_{m+1}+\nu_{0} u_{m} \quad(m \in \mathbb{Z})
$$

with $k>0$ and given coefficients $\nu_{i}$ with $\nu_{0} \neq 0$. Equations

$$
u_{m}=u_{n}
$$

in unknowns $(m, n) \in \mathbb{Z}^{2}$ have been studied in [2] and [7]. It was shown there that for nondegenerate $\left\{u_{m}\right\},(1.2)$ has only finitely many solutions $m>n \geq 0$. More generally, the equation

$$
u_{m}=v_{n},
$$

where $\left\{u_{m}\right\}$ and $\left\{v_{n}\right\}$ are given recurrence sequences, was treated by Laurent [3] and the current authors [11]. They give a complete qualitative description of the set of solutions $(m, n) \in \mathbb{Z}^{2}$ of (1.3).

It is the purpose of the present paper to derive quantitative results on equations (1.3). Let

$$
P(z)=z^{k}-\nu_{k-1} z^{k-1}-\ldots-\nu_{0}=\prod_{i=1}^{r}\left(z-\alpha_{i}\right)^{\sigma_{i}}
$$

with distinct roots $\alpha_{1}, \ldots, \alpha_{r}$ be the companion polynomial of relation (1.1). It is well known that there exist polynomials $f_{1}, \ldots, f_{r}$ which are not all zero and have $\operatorname{deg} f_{i} \leq \sigma_{i}-1$ such that

$$
u_{m}=\sum_{i=1}^{r} f_{i}(m) \alpha_{i}^{m} .
$$

Notice that in view of (1.4) we have in particular

$$
\operatorname{deg} f_{i}<k \quad(i=1, \ldots, r) .
$$


The sequence $\left\{u_{m}\right\}$ is called nondegenerate if none of the quotients $\alpha_{i} / \alpha_{j}$ for $i \neq j$ is a root of unity.

To unify the notation in the sequel, we will consider instead of (1.5) the function

$$
F(x)=\sum_{i=0}^{r} f_{i}(x) \alpha_{i}^{x}
$$

of polynomial-exponential type, where $\alpha_{0}$ is a root of unity, where $\alpha_{i} / \alpha_{j}$ for $i \neq j$ is not a root of unity, and where the $f_{i}$ are polynomials with

$$
\operatorname{deg} f_{i}<k \quad(0 \leq i \leq r)
$$

and with

$$
f_{i} \neq 0 \quad \text { for } i=1, \ldots, r \text {. }
$$

If the companion polynomial (1.4) of $\left\{u_{m}\right\}$ does not have a zero which is a root of unity, then we put $f_{0}(x) \equiv 0$ and $\alpha_{0}=1$.

Similarly, we write $v_{m}=G(m)$ with

$$
G(y)=\sum_{i=0}^{r^{\prime}} g_{i}(y) \beta_{i}^{y},
$$

where again we suppose that the $g_{i}$ are polynomials with

$$
\begin{gathered}
\operatorname{deg} g_{i}<k \quad\left(i=0, \ldots, r^{\prime}\right), \\
g_{i} \neq 0 \quad \text { for } i=1, \ldots, r^{\prime}
\end{gathered}
$$

and where we assume, moreover, that $\beta_{i} \neq 0$, that $\beta_{0}$ is a root of unity, and $\beta_{i} / \beta_{j}$ for $i \neq j$ is not a root of unity.

We will suppose throughout that both $r, r^{\prime} \geq 1$. Thus, equation (1.3) becomes

$$
F(x)=G(y),
$$

to be solved in integers $x, y$.

In this paper we will study (1.13) assuming that $F$ and $G$ are defined over the algebraic numbers. So, let $K$ be a number field of degree

$$
[K: \mathbb{Q}]=d
$$

containing $\alpha_{0}, \ldots, \alpha_{r}, \beta_{0}, \ldots, \beta_{r^{\prime}}$. We assume, moreover, that the leading coefficients and the zeros of the polynomials $f_{i}$ and $g_{i}$ are contained in $K$.

We recall the following definitions from [11].

Definition. $F$ and $G$ are called related if

(i) $r=r^{\prime}$,

(ii) either $f_{0} \equiv g_{0} \equiv 0$ or $f_{0} g_{0} \not \equiv 0$, 
(iii) there is a reordering of $\beta_{1}, \ldots, \beta_{r}$ such that

$$
\alpha_{i}^{p}=\beta_{i}^{q} \quad(i=1, \ldots, r)
$$

with certain nonzero integers $p, q$.

Now, suppose that $F$ and $G$ are related with (1.15) and that $r$ is even. $F$ and $G$ are called doubly related if after reordering we have both (1.15) and

$$
\alpha_{i}^{p^{\prime}}=\beta_{i+1}^{q^{\prime}}, \quad \alpha_{i+1}^{p^{\prime}}=\beta_{i}^{q^{\prime}} \quad \text { for } 1 \leq i<r, i \text { odd },
$$

with certain nonzero integers $p^{\prime}, q^{\prime}$.

In the sequel, when $F$ and $G$ are related or doubly related, we will assume that the reorderings guaranteeing (1.15), or (1.15) and (1.16), have been applied.

It was shown in [11] that the related pair $F, G$ is doubly related if and only if

$$
\alpha_{i} \alpha_{i+1} \text { and } \beta_{i} \beta_{i+1} \text { for } 0 \leq i<r, i \text { odd, are roots of unity. }
$$

Moreover, if $p, q$ and $p^{\prime}, q^{\prime}$ are as in (1.15) and (1.16), then $p / q=-p^{\prime} / q^{\prime}$. There cannot be a third permutation with a property like (1.15) or (1.16).

A pair $F, G$ that is related but not doubly related is called simply related. Let us mention in this context that relatedness as well as double relatedness, in view of Lemma 6.3 below, are decidable properties.

We denote by $M(K)$ the set of absolute values of $K$ and by $M_{\infty}(K)$ the subset of archimedean absolute values in $M(K)$. Let $S$ be the subset of $M(K)$ consisting of $M_{\infty}(K)$ and those absolute values \|\|$_{v}$ in $M(K)$ for which $\left\|\alpha_{i}\right\|_{v} \neq 1$ or $\left\|\beta_{j}\right\|_{v} \neq 1$ for some $i$ with $1 \leq i \leq r$ or some $j$ with $1 \leq j \leq r^{\prime}$. It is clear that the cardinality $s$ of $S$ is finite and has

$$
s \leq d+\omega,
$$

where $\omega$ denotes the number of distinct prime ideals occurring in the decomposition of the fractional ideals $\left(\alpha_{i}\right),\left(\beta_{j}\right)$ in $K$.

THEOREM 1. Suppose that $F$ and $G$ are not related. Then equation (1.13) has not more than

$$
2^{s^{7} 2^{45 d !\left(k^{2}\left(r+r^{\prime}+2\right)\right) !}}
$$

solutions $(x, y) \in \mathbb{Z}^{2}$.

Now, suppose that $F$ and $G$ are related. In [11] (Proposition 1) we proved the following:

When $F$ and $G$ are simply related, then all but finitely many solutions of (1.13) satisfy the system of equations

$$
f_{i}(x) \alpha_{i}^{x}=g_{i}(y) \beta_{i}^{y} \quad(i=0, \ldots, r) .
$$


When $F$ and $G$ are doubly related, then all but finitely many solutions of (1.13) satisfy either (1.20) or the system

(1.21a) $f_{i}(x) \alpha_{i}^{x}=g_{i+1}(y) \beta_{i+1}^{y}, \quad f_{i+1}(x) \alpha_{i+1}^{x}=g_{i}(y) \beta_{i}^{y} \quad(1 \leq i<r, i$ odd $)$,

$$
f_{0}(x) \alpha_{0}^{x}=g_{0}(y) \beta_{0}^{y} .
$$

If $F$ and $G$ are simply related, we write $\mathfrak{S}$ for the set of solutions $(x, y) \in$ $\mathbb{Z}^{2}$ of (1.13) that do not satisfy the system (1.20).

If $F$ and $G$ are doubly related, we let $\mathfrak{S}$ be the set of solutions of (1.13) which satisfy neither (1.20) nor (1.21a,b).

TheOrem 2. Suppose that $F$ and $G$ are related. Then $\mathfrak{S}$ has cardinality

$$
|\mathfrak{S}| \leq 2^{s^{7} 2^{45 d !\left(2 k^{2}(r+1)\right) !}} .
$$

Remark. If in (1.7) and (1.10), $f_{0}=g_{0}=0$, then in (1.22), $r+1$ may be replaced by $r$. The significant feature in Theorems 1 and 2 is that the bounds (1.19) and (1.22) are uniform, as they involve only the degree $d$ of the field $K$, the bound $k$ for the degrees of the polynomials $f_{i}$ and $g_{j}$, the numbers $r$ and $r^{\prime}$ of characteristic roots and the number $s$ of absolute values in $S$. No particular care was taken in optimizing the actual shape of the bounds; we rather tried to avoid painstaking estimates.

For $F$ and $G$ related, we still want some information about the solutions $(x, y) \in \mathbb{Z}^{2}$ of either (1.20) or of (1.20) and (1.21a,b).

Again following [11], we call the ordered pair $F, G$ exceptional if

(i) $F$ and $G$ are simply related,

(ii) there is a natural number $N>1$ which is an integral power of each $\alpha_{i}$ and each $\beta_{i}$ with $1 \leq i \leq r$,

(iii) either $\left|\alpha_{i}\right|>1$ for $1 \leq i \leq r$, or $\left|\alpha_{i}\right|<1$ for $1 \leq i \leq r$,

(iv) $f_{0}$ and $g_{0}$ are constant,

(v) each $g_{i}$ is constant and for $1 \leq i \leq r, f_{i}(x)=a_{i}(x-A)^{l_{i}}$ where $A$ is rational and $l_{i}>0$.

We remark that by Lemma 6.3 below, exceptionality is decidable. Notice that if $F, G$ is exceptional, then by (1.15) either $\left|\beta_{i}\right|>1$ for $1 \leq i \leq r$ or $\left|\beta_{i}\right|<1$ for $1 \leq i \leq r$. Thus, in view of (1.17), if the pair $F, G$ is doubly related neither $F, G$ nor $G, F$ is exceptional.

If $F$ and $G$ are related, then as $\alpha_{0}$ and $\beta_{0}$ are roots of unity, we may extend (1.15) also to $i=0$ (by replacing $p, q$ by suitable multiples, if necessary). So we will suppose in the sequel that we have

$$
\alpha_{i}^{p}=\beta_{i}^{q} \quad \text { for } i=0, \ldots, r
$$

with nonzero integers $p, q$. And in fact, we will assume that $p>0$ in (1.23), and $p$ is chosen minimal with this property. 
Similarly, when $F$ and $G$ are doubly related we will assume instead of (1.16) that

$$
\begin{array}{r}
\alpha_{i}^{p^{\prime}}=\beta_{i+1}^{q^{\prime}}, \quad \alpha_{i+1}^{p^{\prime}}=\beta_{i}^{q^{\prime}} \quad \text { for } i=1, \ldots, r, i \text { odd, } \\
\text { and } \alpha_{0}^{p^{\prime}}=\beta_{0}^{q^{\prime}},
\end{array}
$$

with nonzero integers $p^{\prime}, q^{\prime}$, where again $p^{\prime}>0, p^{\prime}$ minimal.

The systems $(1.20)$ or $(1.21 \mathrm{a}, \mathrm{b})$ are of exactly the same type. Thus it suffices to deal with (1.20).

Suppose that the polynomials $f_{i}$ in (1.7) have leading coefficients $a_{i}$ and zeros $A_{1}^{(i)}, \ldots, A_{l_{i}}^{(i)}(i=1, \ldots, r)$, and similarly define $b_{j}$ and $B_{1}^{(j)}, \ldots, B_{m_{j}}^{(j)}$ with respect to the polynomials $g_{j}$ in (1.10). Write

$$
H(F)=\max \left\{h\left(a_{i}\right), h\left(\alpha_{i}\right), h\left(A_{j}^{(i)}\right) \text { over } 0 \leq i \leq r, 1 \leq j \leq l_{i}\right\},
$$

where $h$ is the absolute height whose definition will be detailed in Section 5 . Similarly define $H(G)$ with respect to $G$.

Let $H$ be a quantity having

$$
H \geq \max \{3, H(F), H(G)\} .
$$

We write $c(d, k, H)$ for an unspecified but effective constant that depends only upon $d, k$ and $H$. So $c(d, k, H)$ at different instances may have different values.

In Sections 8-11 we will detail some arguments that may give the reader an idea about explicit versions of these constants.

Theorem 3. Suppose that $F$ and $G$ are related, but neither $F, G$ nor $G, F$ is exceptional. Then it is decidable whether (1.20) admits infinitely many solutions $(x, y) \in \mathbb{Z}^{2}$ or not.

(a) If (1.20) has only finitely many solutions, then all but at most

$$
M_{1}:=2^{s^{7} 2^{540 d !(2 k) !}}
$$

solutions satisfy

$$
\max (|x|,|y|) \leq c(d, k, H),
$$

where $c(d, k, H)$ is effectively computable.

(b) If (1.20) has infinitely many solutions, there is a unique linear oneparameter family of solutions

$$
x(t)=p t+p^{\prime}, \quad y(t)=q t+q^{\prime} \quad(t \in \mathbb{Z})
$$

with $0 \leq p^{\prime}<p$ such that all but at most $M_{1}$ solutions lie in this family or satisfy (1.27).

The family (1.28) is effectively computable. 
The point of the theorem is that the solutions with (1.27) as well as the family (1.28) may be effectively computed, while on the other hand $M_{1}$ is independent of $H$.

R e m a r k. The family (1.28) occurs if and only if we have the polynomial identities

$$
f_{i}\left(p X+p^{\prime}\right) \alpha_{i}^{p^{\prime}} \equiv g_{i}\left(q X+q^{\prime}\right) \beta_{i}^{q^{\prime}} \quad(0 \leq i \leq r) .
$$

Theorem 4. Suppose that the pair F, $G$ is exceptional. Then it is decidable whether (1.20) admits infinitely many solutions $(x, y) \in \mathbb{Z}^{2}$ or not.

(a) If (1.20) has only finitely many solutions, then all but at most

$$
M_{2}:=(r-1) 2^{2^{1080} s^{7}}
$$

solutions satisfy

$$
\max (|x|,|y|) \leq c(d, k, H) .
$$

(b) If (1.20) has infinitely many solutions, then there is a finite number of one-parameter exponential families of solutions of the type

$$
x_{j}(t)=p E_{j} R^{t}+A, \quad y_{j}(t)=q E_{j} R^{t}+F t+G_{j} \quad(t \in \mathbb{Z}, t \geq 0)
$$

with $1 \leq j \leq c(d, k, r, H)$ such that all but at most $M_{2}$ solutions lie in one of these families or satisfy (1.31). The parameters $p, q, E_{j}, R, A, F, G_{j}$ determining the families are effectively computable. Moreover, in (1.32), $R$ is an integral power of each $\alpha_{1}, \ldots, \alpha_{r}$ and $F$ is a nonzero integer that does not depend upon the particular family.

Theorems 1-4 imply in particular that for equations $u_{m}=v_{n}$ we have an algorithm that allows us to determine effectively all solutions, except possibly a finite set whose cardinality is uniformly bounded.

A particular instance of our results concerns equations $u_{m}=u_{n}$ or more generally

$$
a u_{m}=b u_{n}
$$

with $a, b \in K^{*}$, where $\left\{u_{m}\right\}_{m \in \mathbb{Z}}$ is a nondegenerate linear recurrence sequence as in (1.1). We suppose that relation (1.1) is minimal, i.e., that $u_{m}$ does not satisfy a relation of smaller order. Moreover, we assume that $k>1$ and that at least one of the characteristic roots of the polynomial in (1.4) is not a root of unity. Using the representation (1.5), we may write $u_{m}=F(m)$ with $F$ as in (1.7), and here by (1.4), if $f_{0} \equiv 0$ then $r \leq k$ and otherwise $r+1 \leq k$. We suppose that $\left\{u_{m}\right\}$, i.e., $F(m)$, is defined over $K$. It is clear that $F$ is related with itself. We call $\left\{u_{m}\right\}$ symmetric if $F$ is doubly related with itself.

The relations (1.15) or (1.23) now simply reduce to $\alpha_{i}^{1}=\alpha_{i}^{1}(i=$ $0, \ldots, r)$, and if $F$ is symmetric, then since in (1.15), (1.16) we have $p / q=$ 
$-p^{\prime} / q^{\prime}$, the relations (1.16) or (1.24) become

$$
\alpha_{i}^{w}=\alpha_{i+1}^{-w} \quad \text { for } 1 \leq i<r, i \text { odd, }
$$

where $w>0$. In fact, it follows from Lemma 6.1 below that here the minimal $w$ has

$$
0<w \leq 2 d^{2} .
$$

Let $H_{1}=\max \{3, H(F)\}$.

COROLlary 1. (i) Suppose that $\left\{u_{m}\right\}$ is not symmetric. Then all but at most

$$
M_{3}:=2^{s^{7} 2^{46 d !\left(2 k^{3}\right) !}}
$$

solutions $(m, n) \in \mathbb{Z}^{2}$ of the equation

$$
u_{m}=u_{n}
$$

with $m \neq n$ satisfy

$$
\max \{|m|,|n|\} \leq c\left(d, k, H_{1}\right),
$$

where $c\left(d, k, H_{1}\right)$ is effective.

(ii) If $\left\{u_{m}\right\}$ is symmetric, then in addition to solutions as in (i), there may be a one-parameter family of solutions

$$
m(t)=w t+w^{\prime}, \quad n(t)=-w t+w^{\prime \prime} \quad(t \in \mathbb{Z})
$$

where $w$ is an integer with $0<w \leq 2 d^{2}$, and $w^{\prime}$ has $0 \leq w^{\prime}<w$. The family (1.39) is effectively computable.

We next treat the slightly more general equation

$$
a u_{m}=b u_{n} .
$$

Let $H_{2}=\max \{3, H(F), h(b / a)\}$.

COROllary 2. (i) Suppose that $b / a \neq 1$ is a root of unity. Then, apart from at most

$$
M_{4}:=2^{s^{7} 2^{46 d !\left(2 k^{3}\right) !}}
$$

solutions, and apart from a possible one-parameter family as in (1.39), the solutions $(m, n) \in \mathbb{Z}^{2}$ of (1.40) satisfy

$$
\max \{|m|,|n|\} \leq c\left(d, k, H_{2}\right) .
$$

Again the family is effectively computable and the constant in (1.42) is effective. The family (1.39) may only occur if $\left\{u_{m}\right\}$ is symmetric.

(ii) Suppose that $a / b$ is not a root of unity. Then all but at most $M_{4}$ solutions of (1.40) have (1.42).

A qualitative version of Corollaries 1 and 2 was proved by Laurent [4] (Théorème 2). 
2. Polynomial-exponential equations. We consider equations

$$
\sum_{i=1}^{h} P_{i}(\mathbf{x}) \boldsymbol{\alpha}_{i}^{\mathbf{x}}=0
$$

in variables $\mathbf{x}=\left(x_{1}, \ldots, x_{N}\right) \in \mathbb{Z}^{N}$, where the $P_{i}$ are nonzero polynomials with coefficients in $K$ of total degree $\leq \delta$, and where $\boldsymbol{\alpha}_{i}^{\mathbf{x}}=\alpha_{i 1}^{x_{1}} \ldots \alpha_{i N}^{x_{N}}$. Here we suppose that the $\alpha_{i j}$ are elements in $K^{*}$. The letter $S$ will indicate a finite set $S \subset M(K)$ which contains $M_{\infty}(K)$ as well as the nonarchimedean absolute values $v$ of $K$ for which $\left|\alpha_{i j}\right|_{v} \neq 1$ for some pair $i, j(1 \leq i \leq h$, $1 \leq j \leq N)$. We set $s=\operatorname{card} S$.

When $\mathcal{P}$ is a partition of $\{1, \ldots, h\}$ and $\pi$ is a subset of $\{1, \ldots, h\}$, we write $\pi \in \mathcal{P}$ if $\pi$ is among the subsets belonging to $\mathcal{P}$. Consider the splitting of equation (2.1) into the system

$$
\sum_{i \in \pi} P_{i}(\mathbf{x}) \boldsymbol{\alpha}_{i}^{\mathbf{x}}=0 \quad(\pi \in \mathcal{P}) .
$$

We denote by $\mathfrak{S}(\mathcal{P})$ the set of solutions $\mathbf{x} \in \mathbb{Z}^{N}$ of $(2.1 \mathcal{P})$ which do not satisfy a system $(2.1 \mathcal{Q})$ for a proper refinement $\mathcal{Q}$ of $\mathcal{P}$. It is clear that any solution $\mathbf{x} \in \mathbb{Z}^{N}$ of $(2.1)$ is contained in some set $\mathfrak{S}(\mathcal{P})$ for a suitable $\mathcal{P}$ (which is not necessarily unique). Thus, to give an upper bound for the number of solutions of (2.1), it suffices to give an upper bound for the cardinalities of the sets $\mathfrak{S}(\mathcal{P})$ where $\mathcal{P}$ runs through the partitions of $\{1, \ldots, h\}$.

Given $\mathcal{P}$ and elements $i, j \in\{1, \ldots, h\}$, we write $i \stackrel{\mathcal{P}}{\sim} j$ if $i, j$ belong to the same subset $\pi \in \mathcal{P}$. We denote by $G(\mathcal{P})$ the subgroup of $\mathbb{Z}^{N}$ consisting of points $\mathbf{x}$ having

$$
\boldsymbol{\alpha}_{i}^{\mathbf{x}}=\boldsymbol{\alpha}_{j}^{\mathbf{x}} \quad \text { for every } i, j \text { with } i \stackrel{\mathcal{P}}{\sim} j .
$$

The following theorem is the main result of Schlickewei and Schmidt [10].

Theorem A. Suppose that $G(\mathcal{P})=\{\mathbf{0}\}$. Then

$$
|\mathfrak{S}(\mathcal{P})|<2^{20 N^{4}+N s^{7} 2^{43 d !(D h) !},}
$$

where $D=\left(\begin{array}{c}N+\delta \\ N\end{array}\right)$.

We mention that Theorem A is a consequence of the Subspace Theorem in diophantine approximation.

3. Groups $G(\mathcal{P})$. To prove our theorems we will apply Theorem A of Section 2.

It is clear that equation (1.13) is a special instance of (2.1), where in view of (1.7) and (1.10) we have $h=r+r^{\prime}+2, N=2$ and $\delta<k$. We will symbolize the $r+r^{\prime}+2$ summands of equation (1.13) as

$$
0_{x}, 1_{x}, \ldots, r_{x}, 0_{y}, 1_{y}, \ldots, r_{y}^{\prime} \text {. }
$$


Given a partition $\mathcal{P}$ of $\left\{0_{x}, \ldots, r_{x}, 0_{y}, \ldots, r_{y}^{\prime}\right\}$, the relations (2.2) are

$$
\begin{aligned}
\alpha_{i}^{x}=\alpha_{j}^{x} & \text { for all } i, j \text { with } i_{x} \stackrel{\mathcal{P}}{\sim} j_{x}, \\
\beta_{i}^{y}=\beta_{j}^{y} & \text { for all } i, j \text { with } i_{y} \stackrel{\mathcal{P}}{\sim} j_{y}, \\
\alpha_{i}^{x}=\beta_{j}^{y} & \text { for all } i, j \text { with } i_{x} \stackrel{\mathcal{P}}{\sim} j_{y} .
\end{aligned}
$$

We say that $\mathcal{P}$ contains an essential singleton if $\mathcal{P}$ contains a one-element subset $\left\{i_{x}\right\}$ or $\left\{i_{y}\right\}$ and if, moreover, in the case $\left\{i_{x}\right\}=\left\{0_{x}\right\}$ or $\left\{i_{y}\right\}=\left\{0_{y}\right\}$ we have $f_{0}(x) \not \equiv 0$ or $g_{0}(x) \not \equiv 0$, respectively.

Lemma 3.1. Suppose that $\mathcal{P}$ contains an essential singleton. Then

$$
|\mathfrak{S}(\mathcal{P})| \leq 2^{s^{7} 2^{44 d !\left(k\left(r+r^{\prime}+2\right)\right) !}} .
$$

Pr o o f. Suppose without loss of generality that $\left\{i_{y}\right\} \in \mathcal{P}$. Then $(1.13 \mathcal{P})$ contains the equation $g_{i}(y) \beta_{i}^{y}=0$. As $g_{i}$ is a nonzero polynomial of degree $\leq k$, this equation has not more than $k$ solutions, say $y_{1}, \ldots, y_{k} \in \mathbb{Z}$. Given $y$, we write $G(y)=c$ and equation (1.13) becomes

$$
f_{0}(x) \alpha_{0}^{x}+f_{1}(x) \alpha_{1}^{x}+\ldots+f_{r}(x) \alpha_{r}^{x}-c \cdot 1^{x}=0 .
$$

Consider partitions $\mathcal{Q}$ of the set $\{0,1, \ldots, r, r+1\}$, where $r+1$ symbolizes the summand $-c \cdot 1^{x}$ in (3.5).

Either, $\mathcal{Q}$ contains a singleton $i$ with $1 \leq i \leq r$. Then $f_{i}(x) \alpha_{i}^{x}=0$, and there are not more than $k$ solutions $x \in \mathbb{Z}$. Consequently, in this case

$$
|\mathfrak{S}(\mathcal{P})| \leq k^{2} .
$$

Or, for each $i(1 \leq i \leq r)$ there exists $j$ with $0 \leq j \leq r+1$ and $j \neq i$ such that $i \sim j$. But then $G(\mathcal{Q})$ is contained in the set of $x \in \mathbb{Z}$ having

$$
\alpha_{i}^{x}=\alpha_{j}^{x} .
$$

As we assume that $F(x)$ is nondegenerate, (3.7) has only the trivial solution $x=0$. Hence $G(\mathcal{Q})=\{0\}$. Thus we may apply Theorem A to (3.5), and consequently, $(3.5 \mathcal{Q})$ has not more than

$$
2^{20+s^{7} 2^{43 d !(k(r+2)) !}}
$$

solutions.

Allowing a factor $k$ for the number of possible values $y$ and a factor $2^{(r+2)^{2}}$ for the number of possible partitions $\mathcal{Q}$ we may conclude from (3.8) that

$$
|\mathfrak{S}(\mathcal{P})| \leq k \cdot 2^{(r+2)^{2}} \cdot 2^{20+s^{7} 2^{43 d !(k(r+2)) !}} \leq 2^{s^{7} 2^{44 d !\left(k\left(r+r^{\prime}+2\right)\right) !} .}
$$

It is clear that we obtain the same estimate if $\mathcal{P}$ contains an essential singleton $\left\{i_{x}\right\}$, and in view of (3.6) the lemma follows. 
LEMmA 3.2. Let $\mathcal{P}$ be a partition of $\left\{0_{x}, \ldots, r_{x}, 0_{y}, \ldots, r_{y}^{\prime}\right\}$ with $G(\mathcal{P})=$ $\{\mathbf{0}\}$. Then

$$
|\mathfrak{S}(\mathcal{P})| \leq 2^{s^{7} 2^{44 d !\left(k^{2}\left(r+r^{\prime}+2\right)\right) !} .}
$$

Proof. We apply Theorem A with $h=r+r^{\prime}+2, N=2$ and $\delta<k$ to obtain, from (2.3),

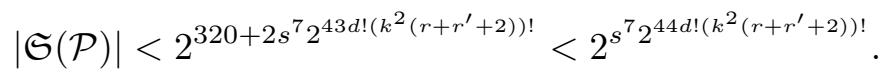

Lemma 3.3. Suppose that $F$ and $G$ are not related. Let $\mathcal{P}$ be a partition of $\left\{0_{x}, 1_{x}, \ldots, r_{x}, 0_{y}, 1_{y}, \ldots, r_{y}^{\prime}\right\}$. Then either $\mathcal{P}$ contains an essential singleton or $G(\mathcal{P})=\{(0,0)\}$.

Proof. See Laurent [3], Lemme 2, and [11], §5.

Lemma 3.4. Suppose that $F$ and $G$ are related and $f_{0}(x) g_{0}(x) \not \equiv 0$. Let $\mathcal{P}$ be a partition of $\left\{0_{x}, \ldots, r_{x}, 0_{y}, \ldots, r_{y}\right\}$ that does not contain an essential singleton and such that $G(\mathcal{P}) \neq\{(0,0)\}$.

(i) If $F$ and $G$ are simply related, then

$$
\mathcal{P}=\left\{0_{x}, 0_{y}\right\},\left\{1_{x}, 1_{y}\right\}, \ldots,\left\{r_{x}, r_{y}\right\} .
$$

(ii) If $F$ and $G$ are doubly related, then either $\mathcal{P}$ is as in (3.11) or

$$
\mathcal{P}=\left\{0_{x}, 0_{y}\right\},\left\{1_{x}, 2_{y}\right\},\left\{2_{x}, 1_{y}\right\}, \ldots,\left\{(r-1)_{x}, r_{y}\right\},\left\{r_{x},(r-1)_{y}\right\} .
$$

Proof. See [3], Lemme 2, and [11], §5.

4. Proof of Theorems 1 and 2. As for Theorem 1, in view of Lemma 3.3 we have only partitions $\mathcal{P}$ of $\left\{0_{x}, \ldots, r_{x}, 0_{y}, \ldots, r_{y}^{\prime}\right\}$ that have $G(\mathcal{P})=$ $\{(0,0)\}$ or contain an essential singleton. In Theorem 2 the situation is the same by the definition of $\mathfrak{S}$. Therefore, we may apply Lemmata 3.1 and 3.2. Comparing (3.4) and (3.10) we see that for any partition $\mathcal{P}$ under consideration we have

$$
|\mathfrak{S}(\mathcal{P})| \leq 2^{s^{7} 2^{44 d !\left(k^{2}\left(r+r^{\prime}+2\right)\right) !}} .
$$

As the number of partitions of $\left\{0_{x}, \ldots, r_{x}, 0_{y}, \ldots, r_{y}^{\prime}\right\}$ to be considered does not exceed $2^{2\left(r+r^{\prime}+2\right)}$, we get

$$
|\mathfrak{S}| \leq 2^{s^{7} 2^{45 d !\left(k^{2}\left(r+r^{\prime}+2\right)\right) !},}
$$

and Theorem 1 follows. If $F$ and $G$ are related, then $r=r^{\prime}$ and hence (4.1) implies Theorem 2 as well.

5. Heights. Recall that $K$ is a number field of degree $d$. We denote by $M(K)$ an indexing set for the absolute values of $K$. Thus given $v \in$ 
$M(K),||_{v}$ is an extension of either the standard absolute value on $\mathbb{Q}$ or of a $p$-adic absolute value. Moreover, for $\alpha \in K$ and $v \in M(K)$ we define

$$
\|\alpha\|_{v}=|\alpha|_{v}^{d_{v} / d}
$$

where $d_{v}$ denotes the local degree. Given an element $\alpha \in K^{*}$, we put

$$
h(\alpha)=\prod_{v \in M(K)} \max \left\{1,\|\alpha\|_{v}\right\} .
$$

If $\boldsymbol{\alpha}=\left(\alpha_{1}, \ldots, \alpha_{n}\right)$ is a vector in $K^{n}$, we define $\|\boldsymbol{\alpha}\|_{v}=\max \left\{\left\|\alpha_{1}\right\|_{v}, \ldots\right.$ $\left.\ldots,\left\|\alpha_{n}\right\|_{v}\right\}$ and put

$$
H(\boldsymbol{\alpha})=\prod_{v \in M(K)}\|\boldsymbol{\alpha}\|_{v}
$$

Thus, for $\alpha \in K^{*}$ we have $h(\alpha)=H(1, \alpha)$. Given a subset $S$ of $M(K)$, we put

$$
H_{S}(\boldsymbol{\alpha})=\prod_{v \in S}\|\boldsymbol{\alpha}\|_{v}
$$

Lemma 5.1. Given $\alpha_{1}, \ldots, \alpha_{l} \in K^{*}$ we have

$$
\begin{aligned}
h\left(\alpha_{1} \ldots \alpha_{l}\right) & \leq h\left(\alpha_{1}\right) \ldots h\left(\alpha_{l}\right), \\
h\left(\alpha_{1}+\ldots+\alpha_{l}\right) & \leq l h\left(\alpha_{1}\right) \ldots h\left(\alpha_{l}\right) .
\end{aligned}
$$

Moreover, for $\alpha \in K^{*}$ let $C$ be a denominator of $\alpha$, i.e., a natural number such that $C \alpha$ is an algebraic integer. Then $C$ may be chosen such that

$$
h(C) \leq h(\alpha)^{d} .
$$

This is well known. A proof may be found, e.g., in Schmidt [12].

Lemma 5.2 (Dobrowolski [1]). Suppose that $A \in K^{*}$ is not a root of unity. Then

$$
h(A)>1+\frac{1}{20 d^{3}} .
$$

Lemma 5.3. Let $x^{\prime}$ and $y^{\prime}$ be integers. Let $A^{\prime}$ and $B^{\prime}$ be elements in $K^{*}$ such that

$$
x^{\prime}-y^{\prime}-A^{\prime}+B^{\prime} \neq 0 .
$$

Suppose that $H^{\prime}$ is a quantity with

$$
H^{\prime} \geq \max \left\{h\left(A^{\prime}\right), h\left(B^{\prime}\right)\right\} .
$$

Suppose moreover that

$$
\max \left\{\left|x^{\prime}\right|,\left|y^{\prime}\right|\right\} \geq 16 H^{\prime d+3} .
$$

Then

$$
H\left(\left(x^{\prime}-A^{\prime}, y^{\prime}-B^{\prime}, x^{\prime}-y^{\prime}-A^{\prime}+B^{\prime}\right)\right) \geq \frac{\max \left\{\left|x^{\prime}\right|,\left|y^{\prime}\right|\right\}^{1 / 2}}{\max \left\{1,\left|x^{\prime}-y^{\prime}\right|\right\}}
$$


Proof. We may assume that $\left|x^{\prime}\right| \geq\left|y^{\prime}\right|$ and get by (5.2), (5.8),

$$
\begin{aligned}
H\left(\left(x^{\prime}-A^{\prime}, y^{\prime}-B^{\prime}, x^{\prime}-y^{\prime}-A^{\prime}+B^{\prime}\right)\right) & \\
& =\prod_{v \in M(K)} \max \left\{\left\|x^{\prime}-A^{\prime}\right\|_{v},\left\|y^{\prime}-B^{\prime}\right\|_{v},\left\|x^{\prime}-y^{\prime}-A^{\prime}+B^{\prime}\right\|_{v}\right\} \\
& \geq \prod_{v \mid \infty}\left\|x^{\prime}-A^{\prime}\right\|_{v} \prod_{v \nmid \infty}\left\|x^{\prime}-y^{\prime}-A^{\prime}+B^{\prime}\right\|_{v} \\
& =\prod_{v \mid \infty}\left\|x^{\prime}-A^{\prime}\right\|_{v}\left\|x^{\prime}-y^{\prime}-A^{\prime}+B^{\prime}\right\|_{v}^{-1} .
\end{aligned}
$$

On the other hand, by (5.1) and (5.5),

$$
\begin{aligned}
\prod_{v \mid \infty}\left\|x^{\prime}-y^{\prime}-A^{\prime}+B^{\prime}\right\|_{v} & \leq \prod_{v \in M(K)} \max \left\{1,\left\|x^{\prime}-y^{\prime}-A^{\prime}+B^{\prime}\right\|_{v}\right\} \\
& =h\left(x^{\prime}-y^{\prime}-A^{\prime}+B^{\prime}\right) \\
& \leq 3 \max \left\{1,\left|x^{\prime}-y^{\prime}\right|\right\} h\left(A^{\prime}\right) h\left(B^{\prime}\right) .
\end{aligned}
$$

We denote by $\sigma_{1}, \ldots, \sigma_{d}$ the isomorphic embeddings of $K$ in $\mathbb{C}$. Then, in view of (5.10),

$$
\begin{aligned}
\prod_{v \mid \infty}\left\|x^{\prime}-A^{\prime}\right\|_{v} & =\left(\prod_{i=1}^{d}\left|x^{\prime}-\sigma_{i} A^{\prime}\right|\right)^{1 / d} \geq \prod_{i=1}^{d}\left(\left|x^{\prime}\right|-\left|\sigma_{i} A^{\prime}\right|\right)^{1 / d} \\
& \geq \prod_{i=1}^{d}\left(\left|x^{\prime}\right|-h\left(A^{\prime}\right)^{d}\right)^{1 / d}=\left|x^{\prime}\right|-h\left(A^{\prime}\right)^{d} \geq \frac{3}{4}\left|x^{\prime}\right| .
\end{aligned}
$$

Altogether, using (5.9) and (5.10) we get

$$
\begin{aligned}
H\left(\left(x^{\prime}-A^{\prime}, y^{\prime}\right.\right. & \left.\left.-B^{\prime}, x^{\prime}-y^{\prime}-A^{\prime}+B^{\prime}\right)\right) \\
& \geq \frac{3}{4}\left|x^{\prime}\right|\left(3 \max \left\{1,\left|x^{\prime}-y^{\prime}\right|\right\} h\left(A^{\prime}\right) h\left(B^{\prime}\right)\right)^{-1} \\
& \geq \frac{\left|x^{\prime}\right|^{1 / 2}}{\max \left\{1,\left|x^{\prime}-y^{\prime}\right|\right\}}=\frac{\max \left\{\left|x^{\prime}\right|,\left|y^{\prime}\right|\right\}^{1 / 2}}{\max \left\{1,\left|x^{\prime}-y^{\prime}\right|\right\}} .
\end{aligned}
$$

Let

$$
f^{*}(x)=a^{*} \prod_{i=1}^{l}\left(x-A_{i}^{*}\right), \quad a^{*} \neq 0,
$$

and

$$
g^{*}(x)=b^{*} \prod_{j=1}^{m}\left(x-B_{j}^{*}\right), \quad b^{*} \neq 0,
$$

be polynomials with $a^{*}, A_{1}^{*}, \ldots, A_{l}^{*}, b^{*}, B_{1}^{*}, \ldots, B_{m}^{*} \in K$. Suppose that

$$
\max \{l, m\}<k \text {. }
$$


Let $\alpha, \beta \in K^{*}$ be elements with

$$
\|\beta\|_{v}=\|\alpha\|_{v}=1 \quad \text { for each } v \notin S,
$$

where $S$ is as in Section 1 . Thus $\alpha, \beta$ are "S-units".

Lemma 5.4. Let $f(x), g(x), \alpha, \beta$ be as in (5.12)-(5.15). Suppose that

$$
l>0, \quad m>0 \text {. }
$$

Let $H^{*}$ be a quantity with

$$
H^{*} \geq \max \left\{h\left(a^{*}\right), h\left(A_{1}^{*}\right), \ldots, h\left(A_{l}^{*}\right), h\left(b^{*}\right), h\left(B_{1}^{*}\right), \ldots, h\left(B_{m}^{*}\right)\right\} .
$$

Let $x, y, v, w$ be integers such that

$$
\begin{gathered}
f^{*}(x) \alpha^{v}=g^{*}(y) \beta^{w}, \\
f^{*}(x) g^{*}(y) \neq 0,
\end{gathered}
$$

(5.20) $x-A_{i}^{*} \neq y-B_{j}^{*} \quad$ for each pair $i, j(1 \leq i \leq l, 1 \leq j \leq m)$.

Then there exists a pair $i_{0}, j_{0}$ with $1 \leq i_{0} \leq l, 1 \leq j_{0} \leq m$ such that

$$
\begin{aligned}
\prod_{v \in S}\left(\left\|x-A_{i_{0}}^{*}\right\|_{v}\left\|y-B_{j_{0}}^{*}\right\|_{v} \| x\right. & \left.-y-A_{i_{0}}^{*}+B_{j_{0}}^{*} \|_{v}\right) \\
& \leq 3^{4 k^{2}} H^{* 8 k^{2}} \max \{1,|x-y|\}^{4 k^{2}-1} .
\end{aligned}
$$

Proof. (5.15), (5.18) and (5.19) imply

$$
\begin{gathered}
\prod_{v \in S}\left\|f^{*}(x)\right\|_{v}=\prod_{v \in S}\left\|g^{*}(y)\right\|_{v}=\prod_{v \notin S}\left\|f^{*}(x)\right\|_{v}^{-1}=\prod_{v \notin S}\left\|g^{*}(y)\right\|_{v}^{-1}, \\
\left\|f^{*}(x)\right\|_{v}=\left\|g^{*}(y)\right\|_{v} \quad \text { for each } v \notin S .
\end{gathered}
$$

Given $v \in M(K)$, we denote by $\lambda_{v}$ and $\mu_{v}$ respectively subscripts having

$$
\left\|x-A_{\lambda_{v}}^{*}\right\|_{v}=\min _{1 \leq \lambda \leq l}\left\|x-A_{\lambda}^{*}\right\|_{v}, \quad\left\|y-B_{\mu_{v}}^{*}\right\|_{v}=\min _{1 \leq \mu \leq m}\left\|y-B_{\mu}^{*}\right\|_{v} .
$$

We partition the set $M(K) \backslash S$ into two subsets $S_{1}, S_{2}$; here $S_{1}$ consists of those $v$ for which $\left\|y-B_{\mu_{v}}^{*}\right\|_{v} \geq\left\|x-A_{\lambda_{v}}^{*}\right\|_{v}$ and $S_{2}$ of those $v$ for which $\left\|x-A_{\lambda_{v}}^{*}\right\|_{v}>\left\|y-B_{\mu_{v}}^{*}\right\|_{v}$. Then by (5.23),

$$
\begin{array}{ll}
\left\|f^{*}(x)\right\|_{v} \geq\left\|b^{*}\right\|_{v}\left\|y-B_{\mu_{v}}^{*}\right\|_{v}^{m} & \text { for each } v \in S_{1}, \\
\left\|f^{*}(x)\right\|_{v} \geq\left\|a^{*}\right\|_{v}\left\|x-A_{\lambda_{v}}^{*}\right\|_{v}^{l} & \text { for each } v \in S_{2} .
\end{array}
$$

On the other hand, for each $v \notin S$ we have

$$
\left\|x-y-A_{\lambda_{v}}^{*}+B_{\mu_{v}}^{*}\right\|_{v} \leq \max \left\{\left\|x-A_{\lambda_{v}}^{*}\right\|_{v},\left\|y-B_{\mu_{v}}^{*}\right\|_{v}\right\} .
$$

Therefore (5.24) and (5.25) respectively yield

$$
\begin{array}{ll}
\left\|f^{*}(x)\right\|_{v} \geq\left\|b^{*}\right\|_{v}\left\|x-y-A_{\lambda_{v}}^{*}+B_{\mu_{v}}^{*}\right\|_{v}^{m} & \text { for each } v \in S_{1}, \\
\left\|f^{*}(x)\right\|_{v} \geq\left\|a^{*}\right\|_{v}\left\|x-y-A_{\lambda_{v}}^{*}+B_{\mu_{v}}^{*}\right\|_{v}^{l} & \text { for each } v \in S_{2} .
\end{array}
$$


Let $S_{1, \lambda, \mu}$ be the subset of $S_{1}$ for which (5.26) holds with $\left(\lambda_{v}, \mu_{v}\right)=(\lambda, \mu)$. Then

$$
\begin{aligned}
\prod_{v \in S_{1, \lambda, \mu}}\left\|f^{*}(x)\right\|_{v}^{-1} & \leq \prod_{v \in S_{1, \lambda, \mu}}\left\|b^{*}\right\|_{v}^{-1}\left\|x-y-A_{\lambda}^{*}+B_{\mu}^{*}\right\|_{v}^{-m} \\
& =\prod_{v \notin S_{1, \lambda, \mu}}\left\|b^{*}\right\|_{v}\left\|x-y-A_{\lambda}^{*}+B_{\mu}^{*}\right\|_{v}^{m} \\
& \leq \prod_{v \notin S_{1, \lambda, \mu}} \max \left\{1,\left\|b^{*}\right\|_{v}\right\} \max \left\{1,\left\|x-y-A_{\lambda}^{*}+B_{\mu}^{*}\right\|\right\}^{m} \\
& \leq h\left(b^{*}\right) h\left(x-y-A_{\lambda}^{*}+B_{\mu}^{*}\right)^{m} .
\end{aligned}
$$

Taking the product over $1 \leq \lambda \leq l$ and $1 \leq \mu \leq m$ and using the same argument for (5.27), we get

$$
\prod_{v \notin S}\left\|f^{*}(x)\right\|_{v}^{-1} \leq h\left(a^{*}\right)^{l+m} h\left(b^{*}\right)^{l+m} \prod_{\lambda=1}^{l} \prod_{\mu=1}^{m} h\left(x-y-A_{\lambda}^{*}+B_{\mu}^{*}\right)^{l+m},
$$

and thus by (5.17) and Lemma 5.1,

$$
\prod_{v \notin S}\left\|f^{*}(x)\right\|_{v}^{-1} \leq 3^{l m(l+m)} h(x-y)^{l m(l+m)} H^{* 2(l m+1)(l+m)} .
$$

Thus by (5.12) and (5.22) there exists $i_{0}$ with $1 \leq i_{0} \leq l$ such that

$$
\begin{aligned}
\prod_{v \in S}\left\|x-A_{i_{0}}^{*}\right\|_{v} & \leq\left(\prod_{v \notin S}\left\|f^{*}(x)\right\|_{v}^{-1}\right)^{1 / l}\left(\prod_{v \in S}\left\|a^{*}\right\|_{v}\right)^{1 / l} \\
& \leq 3^{m(l+m)} H^{* 2 m(l+m)+l^{-1}(2 l+2 m+1)} \max \{1,|x-y|\}^{m(l+m)} .
\end{aligned}
$$

Similarly, we find $j_{0}$ with $1 \leq j_{0} \leq m$ such that

$$
\prod_{v \in S}\left\|y-B_{j_{0}}^{*}\right\|_{v} \leq 3^{l(l+m)} H^{* 2 l(l+m)+m^{-1}(2 l+2 m+1)} \max \{1,|x-y|\}^{l(l+m)} .
$$

Finally, by (5.14) we get

$$
\begin{aligned}
\prod_{v \in S} \| x & -A_{i_{0}}^{*}\left\|_{v}\right\| y-B_{j_{0}}^{*}\left\|_{v}\right\| x-y-A_{i_{0}}^{*}+B_{j_{0}}^{*} \|_{v} \\
& \leq h\left(x-y-A_{i_{0}}^{*}+B_{j_{0}}^{*}\right) \prod_{v \in S}\left(\left\|x-A_{i_{0}}^{*}\right\|_{v}\left\|y-B_{j_{0}}^{*}\right\|_{v}\right) \\
& \leq 3^{(m+l)^{2}+1} H^{2(m+l)^{2}+(m+l)(2 l+2 m+1) /(m l)+2} \max \{1,|x-y|\}^{(l+m)^{2}+1} \\
& <3^{4 k^{2}} H^{* 8 k^{2}} \max \{1,|x-y|\}^{4 k^{2}-1},
\end{aligned}
$$

as asserted. 


\section{Multiplicative relations}

Lemma 6.1. Let $K$ be a number field of degree $d$. Denote by $w$ the number of roots of unity in $K$. Then

$$
w \leq 2 d^{2} .
$$

Proof. The group of roots of unity in $K$ is cyclic of order $w$. It is generated by a primitive $w$ th root of unity $\zeta$, which is a root of the $w$ th cyclotomic polynomial. Thus $\varphi(w)=\operatorname{deg} \zeta \leq d$, where $\varphi$ is Euler's function. On the other hand, $\varphi(w) \geq(w / 2)^{1 / 2}$, and (6.1) follows.

Lemma 6.2. Suppose again that $K$ has degree $d$. Suppose that $\alpha \in K^{*}$ is such that there exists a natural number $u$ with $\alpha^{u} \in \mathbb{Q}$. Then in fact there exists such a u having

$$
u \leq 2 d^{3} .
$$

Proof. We have $\alpha^{u}=q$ for some $q \in \mathbb{Q}^{*}$ and therefore $\alpha=\zeta|q|^{1 / u}$, where $|q|^{1 / u}$ is the positive $u$ th root of $|q|$ and $\zeta$ is a $2 u$ th root of unity. So $\alpha$ is a root of the polynomial

$$
x^{2 u}-q^{2}=\prod_{\zeta}\left(x-\zeta|q|^{1 / u}\right)
$$

where $\zeta$ runs through the $2 u$ th roots of unity. On the other hand, $\alpha$ is in $K$. Therefore the minimal polynomial of $\alpha$ over $\mathbb{Q}$, say $p(x)$, is of degree say $e \leq d$ and $p(x) \mid\left(x^{2 u}-q^{2}\right)$. Hence $p(x)=\prod_{i=1}^{e}\left(x-\zeta_{i}|q|^{1 / u}\right)$, where $\zeta_{1}, \ldots, \zeta_{e}$ are certain $2 u$ th roots of unity. We may infer that $|q|^{e / u} \prod_{i=1}^{e} \zeta_{i} \in \mathbb{Q}$. This implies that $\prod_{i=1}^{e} \zeta_{i}$ is real, therefore \pm 1 , and therefore $|q|^{e / u} \in \mathbb{Q}$. On the other hand, $\alpha=\zeta_{i}|q|^{1 / u}$ for some $i$. It follows that $\zeta_{i}^{e} \in K$, and hence $\zeta_{i}^{e w}=1$. But this implies that

$$
\alpha^{e w}=\zeta_{i}^{e w}|q|^{e w / u}=|q|^{e w / u} \in \mathbb{Q} .
$$

Consequently, $\alpha^{e w} \in \mathbb{Q}$. In view of Lemma 6.1 and the hypotheses we have

$$
w e \leq 2 d^{3} \text {, }
$$

and the assertion follows.

LEMMA 6.3. Let $\alpha$ and $\beta$ be elements in $K^{*}$, and neither a root of unity. Let $H$ be a quantity with

$$
H \geq \max \{h(\alpha), h(\beta)\} .
$$

Suppose that there exist nonzero integers $z_{1}, z_{2}$ with

$$
\alpha^{z_{1}} \beta^{z_{2}}=1 .
$$

Then there exist such integers having

$$
\max \left\{\left|z_{1}\right|,\left|z_{2}\right|\right\} \leq 60 d^{6} \log H .
$$


Proof. This follows at once from Loxton and van der Poorten [5] (Theorem 3 ). In fact, they proved that there exist nonzero integers $z_{i}$ with (6.4) such that

$$
\max \left\{\left|z_{1}\right|,\left|z_{2}\right|\right\} \leq w \cdot d \cdot \log H \cdot \lambda^{-1},
$$

where $w$ is the number of roots of unity in $K$ and where $\lambda$ is the logarithm of the right hand side in formula (5.7) of Dobrowolski's result as quoted in Lemma 5.2. Thus Lemma 6.1 implies the assertion.

7. Linear equations in $S$-integers. Let $S$ be a finite subset of cardinality $|S|=s$ of $M(K)$ as in Section 1 . We suppose that $S$ contains the set of archimedean prime divisors $M_{\infty}(K)$ of $K$. An element $x \in K$ is called an $S$-integer if $\|x\|_{v} \leq 1$ for each $v \notin S$. We consider the equation

$$
x_{1}+\ldots+x_{n}=0
$$

to be solved in $S$-integers $x_{i} \in K$.

Given a vector $\mathbf{x}=\left(x_{1}, \ldots, x_{n}\right) \in K^{n}$ we define the $S$-height by

$$
H_{S}(\mathbf{x})=\prod_{v \in S}\|\mathbf{x}\|_{v}
$$

with $\|\mathbf{x}\|_{v}$ as in Section 5 .

We will need the following result of Schlickewei [9] (Theorem 1.4).

Lemma 7.1. Suppose that $n \geq 3$. Let $\delta>0$. Then the set of solutions of equation (7.1) in $S$-integers $x_{1}, \ldots, x_{n}$ satisfying

$$
\prod_{v \in S}\left\|x_{1}\right\|_{v} \ldots\left\|x_{n}\right\|_{v}<H_{S}(\mathbf{x})^{1-\delta}
$$

is contained in the union of not more than

$$
t=2 n^{s}\left[(4 s d !)^{2^{37(n-1) d !} s^{6} \delta^{-2}}\right]
$$

proper subspaces $U_{1}, \ldots, U_{t}$ of the $(n-1)$-dimensional linear space $U$ defined by equation (7.1).

8. The equation $f(x) \alpha^{x}=g(y) \beta^{y}$. Let

$$
f(x)=a \prod_{i=1}^{l}\left(x-A_{i}\right) \quad \text { with } a \neq 0
$$

and

$$
g(x)=b \prod_{j=1}^{m}\left(x-B_{j}\right) \quad \text { with } b \neq 0
$$


be polynomials with $a, A_{1}, \ldots, A_{l}, b, B_{1}, \ldots, B_{m} \in K$. We suppose that

$$
\max \{l, m\}<k \text {. }
$$

Assume that $\alpha, \beta \in K^{*}$ are not roots of unity but are multiplicatively dependent. Let $(p, q) \in \mathbb{Z}^{2}$, with $p>0$ minimal, such that

$$
\alpha^{p}=\beta^{q} \quad(=\delta, \text { say }) .
$$

We want to study the solutions $(x, y) \in \mathbb{Z}^{2}$ of the equation

$$
f(x) \alpha^{x}=g(y) \beta^{y} .
$$

In a qualitative sense such equations have been studied in [2] and in [11]. Here we ask for some quantitative information about the solutions.

Throughout, we assume that $H$ is a quantity satisfying

$$
H \geq\left\{3, h(\alpha), h(\beta), h(a), h(b), h\left(A_{1}\right), \ldots, h\left(A_{l}\right), h\left(B_{1}\right), \ldots, h\left(B_{m}\right)\right\} .
$$

Given $(x, y) \in \mathbb{Z}^{2}$ we write

$$
x=p x^{\prime}+p^{\prime}, \quad y=q y^{\prime}+q^{\prime},
$$

where $x^{\prime}, y^{\prime} \in \mathbb{Z}$ and $0 \leq p^{\prime}<p, 0 \leq q^{\prime}<q$. (Notice that $p^{\prime}$ and $q^{\prime}$ are not fixed, but will depend upon $x$ and $y$ respectively.)

LEMMA 8.1. The solutions $(x, y) \in \mathbb{Z}^{2}$ of (8.5) with

$$
\max \{|x|,|y|\} \geq \exp \left(2^{5} d^{6} \log ^{2} H\right)
$$

satisfy

$$
|q x-p y| \leq 2^{19} d k^{15} \log ^{2} H \log (\max \{|q x|,|p y|\}) .
$$

Proof. Using (8.4) and (8.7), we may write (8.5) as

$$
\delta^{x^{\prime}-y^{\prime}}=\frac{g(y)}{f(x)} \alpha^{-p^{\prime}} \beta^{q^{\prime}} .
$$

(We remark in this context that $(8.8)$ implies $f(x) \neq 0$.) Therefore by Lemma $5.1,(8.1)$ and $(8.2)$,

$$
h(\delta)^{\left|x^{\prime}-y^{\prime}\right|} \leq h(a) h(b)\left(\prod_{j=1}^{m} h\left(y-B_{j}\right) \prod_{i=1}^{l} h\left(x-A_{i}\right)\right) h(\alpha)^{\left|p^{\prime}\right|} h(\beta)^{\left|q^{\prime}\right|},
$$

and by (8.6) this is

$$
\begin{aligned}
& \leq H^{2} \cdot 2^{m} \max \{1,|y|\}^{m} H^{m} \cdot 2^{l} \max \{1,|x|\}^{l} H^{l} H^{|p|+|q|} \\
& \leq 2^{2 k} H^{2 k+|p|+|q|} \max \{|x|,|y|\}^{2 k} .
\end{aligned}
$$

But by Lemma 6.3, we have

$$
\max \{|p|,|q|\} \leq 60 d^{6} \log H .
$$


Combination of Lemma 5.2 and (8.8) yields

$$
\begin{aligned}
\mid x^{\prime}- & y^{\prime} \mid \cdot \frac{1}{30 d^{3}} \\
& \leq 2 k \log 2+\left(2 k+120 d^{6} \log H\right) \log H+2 k \log (\max \{|x|,|y|\}) \\
& \leq 4 k \log (\max \{|x|,|y|\}) .
\end{aligned}
$$

Finally, by (8.10) this implies

$$
\begin{aligned}
|q x-p y| & \leq\left|p q x^{\prime}-p q y^{\prime}\right|+\left|q p^{\prime}\right|+\left|p q^{\prime}\right| \\
& \leq 3600 d^{12} \log ^{2} H \cdot 120 d^{3} k \log (\max \{|x|,|y|\})+7200 d^{12} \log ^{2} H \\
& \leq 2^{19} d^{15} k \log ^{2} H \log (\max \{|x|,|y|\}) \\
& \leq 2^{19} d^{15} k \log ^{2} H \log (\max \{|q x|,|p y|\}),
\end{aligned}
$$

as asserted.

9. The equation $f(x) \alpha^{x}=g(y) \beta^{y}$, continued. We assume (8.1)-(8.7) throughout. Recall that our number field $K$ has degree $d$. Let $S$ be the finite subset of $M(K)$ of Section 1 with $|S|=s$ and assume that $\|\alpha\|_{v}=\|\beta\|_{v}=1$ for $v \notin S$.

Lemma 9.1. Let $c_{1}, c_{2}, c_{3}$ be integers with $c_{1} c_{2} \neq 0$ and $c_{1} / c_{2} \neq q / p$. Then the number of common solutions $(x, y) \in \mathbb{Z}^{2}$ of the two equations

$$
c_{1} x=c_{2} y+c_{3}, \quad f(x) \alpha^{x}=g(y) \beta^{y}
$$

does not exceed

$$
2^{s^{7} 2^{44 d !(2 k) !}} .
$$

Proof. We assume without loss of generality that $\left(c_{1}, c_{2}\right)=1$. Then we may parametrize the solutions of the linear equation as $x=x_{0}+c_{2} t$, $y=y_{0}+c_{1} t(t \in \mathbb{Z})$, where $x_{0}$ and $y_{0}$ are fixed and the second equation in (9.1) becomes

$$
f\left(c_{2} t+x_{0}\right) \alpha^{x_{0}}\left(\alpha^{c_{2}}\right)^{t}=g\left(c_{1} t+y_{0}\right) \beta^{y_{0}}\left(\beta^{c_{1}}\right)^{t} \quad(t \in \mathbb{Z}) .
$$

Equation (9.3) is of the type covered by Theorem A of Section 2. We have to consider two partitions. The first corresponds to the vanishing of each side in (9.3) and gives $f\left(c_{2} t+x_{0}\right)=0$. Since $f \not \equiv 0, \operatorname{deg} f<k$, this has less than $k$ solutions.

The second partition of (9.3) has no vanishing term. The corresponding group $G(\mathcal{P})$ is defined by

$$
\left(\alpha^{c_{2}}\right)^{z}=\left(\beta^{c_{1}}\right)^{z} .
$$

As $\alpha$ and $\beta$ are not roots of unity and $c_{1} / c_{2} \neq q / p$, (8.4) implies that (9.4) has only the trivial solution $z=0$. By Theorem $\mathrm{A}$, this partition gives not 
more than

$$
2^{20+s^{7} 2^{43 d !(2 k) !}}
$$

solutions. Since this, plus $k$, does not exceed (9.2), the lemma follows.

Lemma 9.2. Suppose that in (8.1), (8.2) we have

$$
\min \{l, m\}>0 \text {. }
$$

Let $H$ be as in (8.6). Then the number of solutions $(x, y) \in \mathbb{Z}^{2}$ of equation (8.5) satisfying

$$
q x-q A_{i} \neq p y-p B_{j} \quad \text { for each pair } i, j(1 \leq i \leq l, 1 \leq j \leq m)
$$

and

$$
\max \{|x|,|y|\}>\exp \left(2^{10} k^{4} d^{6} \log ^{2} H\right)
$$

does not exceed

$$
2^{s^{7} 2^{45 d !(2 k) !}}-2 d^{2} k^{3} .
$$

Pr o of. We first remark that (9.7) implies that we are only considering solutions $(x, y)$ with $f(x) g(y) \neq 0$. Given a solution $(x, y)$, we consider the product

$$
\prod_{i=1}^{l}\left(x-A_{i}\right) \prod_{j=1}^{m}\left(y-B_{j}\right)
$$

corresponding to $f(x) g(y)$ (but omitting the leading coefficients). We partition the solutions $(x, y)$ into classes $\mathcal{L}=\mathcal{L}(i, j)(1 \leq i \leq l, 1 \leq j \leq m)$ as follows: $(x, y)$ belongs to $\mathcal{L}(i, j)$ if the term $\left(x-A_{i}\right)\left(y-B_{j}\right)$ in $(9.9)$ is small in the sense of (5.21) of Lemma 5.4. Two different classes may overlap, but this is of no importance. We will give an upper bound for the number of solutions in each single class. As the total number of classes is

$$
l m<k^{2},
$$

our assertion (9.8) will eventually follow.

We now restrict ourselves to solutions $x, y$ in a fixed class say $\mathcal{L}\left(i_{0}, j_{0}\right)$, and so we may assume in the sequel that Lemma 5.4 holds true for the pair $\left(i_{0}, j_{0}\right)$. To simplify the notation we will omit subscripts in what follows, and we will write $A, B$ instead of $A_{i_{0}}, B_{j_{0}}$. Recall (8.7), i.e.,

$$
x=p x^{\prime}+p^{\prime}, \quad y=q y^{\prime}+q^{\prime} \quad\left(0 \leq p^{\prime}<p, 0 \leq q^{\prime}<|q|\right) .
$$

Let $D$ be a common denominator of $A$ and $B$. By Lemma 5.1, $D$ may be chosen to satisfy

$$
D \leq H^{2 d}
$$


Put

$$
\begin{gathered}
x_{1}=D q x-D q A, \quad x_{2}=-(D p y-D p B), \\
x_{3}=-(D q x-D p y-D q A+D p B) .
\end{gathered}
$$

The definition of $D$ implies that $x_{1}, x_{2}, x_{3}$ are algebraic integers, and hence in particular they are $S$-integers. Moreover,

$$
x_{1}+x_{2}+x_{3}=0 \text {. }
$$

We want to apply Lemma 7.1 to equation (9.13). To do so, we have to verify hypothesis (7.2). For this purpose we apply Lemma 5.4. We rewrite equation (8.5) as

$$
a D^{-l} q^{-l} \prod_{i=1}^{l}\left(D q x-D q A_{i}\right) \alpha^{x}=b D^{-m} p^{-m} \prod_{j=1}^{m}\left(D p y-D p B_{j}\right) \beta^{y}
$$

and apply Lemma 5.4 with

$$
f^{*}(D q x)=a^{*} \prod_{i=1}^{l}\left(D q x-A_{i}^{*}\right), \quad g^{*}(D p y)=b^{*} \prod_{j=1}^{m}\left(D p y-B_{j}^{*}\right),
$$

where $a^{*}=a D^{-l} q^{-l}, A_{i}^{*}=D q A_{i}, b^{*}=b D^{-m} p^{-m}, B_{j}^{*}=D p B_{j}$. It is clear that $f^{*}(D q x)=f(x), g^{*}(D p y)=g(y)$ and so (8.5) becomes $f^{*}(D q x) \alpha^{x}=$ $g^{*}(D p y) \beta^{y}$. Hypothesis (5.20) of Lemma 5.4 is now hypothesis (9.6).

The conclusion of Lemma 5.4 will be true with

$$
H^{*}=\max \left\{h\left(a^{*}\right), h\left(A_{i}^{*}\right), h\left(b^{*}\right), h\left(B_{j}^{*}\right)\right\} .
$$

By Lemma 6.3 we have (cf. (8.6))

$$
\max \{|p|,|q|\} \leq 60 d^{6} \log H .
$$

Combining (9.15) and (9.11), by Lemma 5.1 we obtain

$$
H^{*} \leq\left(60 d^{6} \log H\right)^{k} H^{1+2 k d} \leq H^{5 k d} .
$$

By Lemma 5.4 we may conclude (using (9.11)) that the $x_{i}$ in (9.12), (9.13) satisfy

$$
\begin{aligned}
\prod_{v \in S}\left\|x_{1}\right\|_{v}\left\|x_{2}\right\|_{v}\left\|x_{3}\right\|_{v} & \leq 3^{4 k^{2}} H^{40 k^{3} d} \max \{1,|D q x-D p y|\}^{4 k^{2}-1} \\
& \leq 3^{4 k^{2}} H^{44 k^{3} d} \max \{1,|q x-p y|\}^{4 k^{2}-1}
\end{aligned}
$$

On the other hand, by Lemma 5.3 and (9.7) we get

$$
\begin{aligned}
& H\left(x_{1}, x_{2}, x_{3}\right) \\
& =H(D q x-D q A, D p y-D p B, D q x-D p y-D q A+D p B) \\
& =H(q x-q A, p y-p B, q x-p y-q A+p B) \geq \frac{\max \{|q x|,|p y|\}^{1 / 2}}{\max \{1,|q x-p y|\}}
\end{aligned}
$$


provided that

$$
\max \{|q x|,|p y|\} \geq 16 \max \{h(q A), h(p B)\}^{d+3} .
$$

By (9.15) we have

$$
16 h(q A)^{d+3} \leq 2^{4}\left(2^{6} d^{6} \log H\right)^{d+3} H^{d+3} \leq\left(2^{3} d H\right)^{6 d+18}
$$

and similarly

$$
16 h(p B)^{d+3} \leq\left(2^{3} d H\right)^{6 d+18} .
$$

Therefore (9.18) will be satisfied if $\max \{|x|,|y|\} \geq\left(2^{3} d H\right)^{6 d+18}$, but this is amply guaranteed by (9.7).

We want to obtain (7.2) with $\delta=1 / 2$. Since $H_{S}\left(x_{1}, x_{2}, x_{3}\right) \geq H\left(x_{1}, x_{2}, x_{3}\right)$, and by (9.16), (9.17), this will be true if we have

$$
\max \{|q x|,|p y|\} \geq 3^{16 k^{2}} H^{176 k^{3} d} \max \{1,|q x-p y|\}^{16 k^{2}} .
$$

We are going to use Lemma 8.1, which is allowed since (9.7) implies (8.8). We obtain

$$
\max \{1,|q x-p y|\} \cdot 3 \cdot H^{11 k d} \leq 2^{21} k d^{15} \log ^{2} H \cdot H^{11 k d} \log (\max \{|q x|,|p y|\}),
$$

and hence (9.19) will be satisfied if we have

$$
2^{21} k d^{15} H^{11 k d} \log ^{2} H \log (\max \{|q x|,|p y|\}) \leq(\max \{|q x|,|p y|\})^{1 / 16 k^{2}} .
$$

But it is easily checked that (9.7) implies (9.20).

So hypothesis (7.2) of Lemma 7.1 is indeed satisfied with $\delta=1 / 2$. We may conclude that the solutions $x_{1}, x_{2}, x_{3}$ of (9.12), (9.13) satisfy one of

$$
2 \cdot 3^{s}(4 s d !)^{2^{74 d !} s^{6} \cdot 4}
$$

linear relations, each of which may be taken as

$$
c_{1} x_{1}+c_{2} x_{2}=0
$$

with $c_{1}, c_{2} \in K,\left(c_{1}, c_{2}\right) \neq(0,0)$.

Suppose first that in (9.22) we have $c_{1}=0$. Then (9.12) implies $y=B$ and therefore $g(B)=0$, which is impossible by (9.7). Similarly, we may conclude that $c_{2} \neq 0$.

Next assume that $c_{1}=c_{2}$. Then (9.22) entails $q x-q A-p y+p B=0$, which is excluded by (9.6).

Therefore, we may assume that in $(9.22), c_{1} c_{2} \neq 0$ and $c_{1} \neq c_{2}$. In view of (9.12), (9.22) is a relation between $x$ and $y$ of the shape

$$
d_{1} x=d_{2} y+d_{3},
$$

and here $d_{1} d_{2} \neq 0$ and $d_{1} / d_{2} \neq q / p$. Now, if a relation (9.23) involves two different solutions $\left(x^{(1)}, y^{(1)}\right),\left(x^{(2)}, y^{(2)}\right)$, then $\left(d_{1}, d_{2}, d_{3}\right)$ is proportional to 
a vector with integral components. Thus we may apply Lemma 9.1. Consequently, each relation (9.23) leads to not more than

$$
2^{s^{7} 2^{44 d !(2 k) !}}
$$

solutions $(x, y)$. Taking into account the number of possible relations (9.23) as estimated in (9.21) and allowing a factor $k^{2}$ from (9.10) for the number of classes $\mathcal{L}$, we finally get the bound

$$
k^{2} \cdot 2 \cdot 3^{s}(4 s d !)^{2^{74 d !} s^{6} \cdot 4} 2^{s^{7} 2^{44 d !(2 k) !}}<2^{s^{7} 2^{45 d !(2 k) !}}-2 d^{2} k^{3} .
$$

The lemma follows.

Lemma 9.3. Let $f$ and $g$ be as in (8.1)-(8.3). Suppose that

$$
\min \{l, m\}>0 \text {. }
$$

Let $i, j$ with $1 \leq i \leq l, 1 \leq j \leq m$ be fixed. Consider the system of two equations

$$
(9.25(i, j)) \quad q x-q A_{i}=p y-p B_{j}
$$

where $q$ and $p$ are as in (8.4), and

$$
f(x) \alpha^{x}=g(y) \beta^{y}
$$

in $(x, y) \in \mathbb{Z}^{2}$. Set

$$
M=2 d^{2} k .
$$

We distinguish two cases.

(a) There exist no integers $p^{\prime}$ and $q^{\prime}$ having

$$
0 \leq p^{\prime}<p, \quad q^{\prime}=B_{j}+\frac{q}{p}\left(p^{\prime}-A_{i}\right)
$$

such that we have the polynomial identity

$$
f\left(p t+p^{\prime}\right) \alpha^{p^{\prime}} \equiv g\left(q t+q^{\prime}\right) \beta^{q^{\prime}} .
$$

Then $(9.25(i, j))$ and $(9.26)$ have at most $M$ common solutions.

(b) There exist unique integers $p^{\prime}, q^{\prime}$ with (9.28) and (9.29). Then the common solutions of $(9.25(i, j))$ and $(9.26)$ consist of the linear one-parameter family

$$
x=p t+p^{\prime}, \quad y=q t+q^{\prime} \quad(t \in \mathbb{Z}),
$$

plus at most $M$ further solutions.

R e m a r k. By Lemma 6.3, $p$ and $q$ in (8.4) satisfy the estimate $\max \{p,|q|\}$ $\leq 60 d^{6} \log H$. Thus by (9.28) it is decidable whether we get a family (9.30) or not. In fact, if there is a solution family (9.30), then its data $p, q, p^{\prime}, q^{\prime}$ are effectively computable (at least in principle). 
Proof of Lemma 9.3. Let $(x, y)$ be a solution of $(9.25(i, j))$. Then writing $x=p t+p^{\prime}$ with $0 \leq p^{\prime}<p$ we get $y=q t+q^{\prime}$ with $q^{\prime}$ as in (9.28). So the solutions of $(9.25(i, j))$ in fact split into families

$$
\mathcal{F}_{p^{\prime}}: \quad x=p t+p^{\prime}, \quad y=q t+q^{\prime}, \quad 0 \leq p^{\prime}<p,
$$

as in (9.30).

Given a family $\mathcal{F}_{p^{\prime}}$, in view of (8.4) equation (9.26) becomes

$$
f\left(p t+p^{\prime}\right) \alpha^{p^{\prime}}=g\left(q t+q^{\prime}\right) \beta^{q^{\prime}} .
$$

Now, if we had two families $\mathcal{F}_{p_{1}^{\prime}}, \mathcal{F}_{p_{2}^{\prime}}$ for which (9.31) is an identity, then comparing the leading coefficients we get $\alpha^{p_{1}^{\prime}} / \beta_{1}^{q_{1}^{\prime}}=\alpha^{p_{2}^{\prime}} / \beta^{q_{2}^{\prime}}$. Hence $\alpha^{p_{1}^{\prime}-p_{2}^{\prime}}=$ $\beta^{q_{1}^{\prime}-q_{2}^{\prime}}$ and by the minimality of $(p, q),\left(p_{1}^{\prime}-p_{2}^{\prime}, q_{1}^{\prime}-q_{2}^{\prime}\right)$ is a multiple of $(p, q)$. In conjunction with $\left|p_{1}^{\prime}-p_{2}^{\prime}\right|<p$ this gives $p_{1}^{\prime}=p_{2}^{\prime}$, whence $q_{1}^{\prime}=q_{2}^{\prime}$. Therefore, if there exist integers $p^{\prime}, q^{\prime}$ with (9.28) and (9.29), then they are uniquely determined.

It remains to be shown that the union of the families $\mathcal{F}_{p^{\prime}}$ for which we do not have an identity (9.29) contains not more than $M$ solutions $(x, y)$, with $M$ as in (9.27). In fact, the solutions $(x, y)$ of $(9.25(i, j))$ satisfy $y=(q / p) x+c$ with $c=B_{j}-(q / p) A_{i}$. Thus (9.26) becomes

$$
f(x) \alpha^{x}=g\left(\frac{q}{p} x+c\right) \beta^{(q / p) x+c} .
$$

Notice that in (9.32) the exponent $(q / p) x+c$ of $\beta$ is an integer; therefore the quotient $\beta^{(q / p) x+c} / \alpha^{x}$ lies in $K$ and so is a $p$ th root of $\beta^{q x+p c} / \alpha^{p x}$ in $K$. But by (8.4), $\beta^{q x}=\alpha^{p x}$. Thus the quotient is a $p$ th root of $\beta^{p c}$ in $K$. Let $\beta^{c}$ be such a $p$ th root. Then (9.32) splits into equations of the shape

$$
f(x)=g\left(\frac{q}{p} x+c\right) \zeta \beta^{c},
$$

where $\zeta$ runs through the $p$ th roots of unity in $K$. Given $\zeta$, either (9.33) holds identically in $x$ or, since $\max \{\operatorname{deg} f, \operatorname{deg} g\}<k$, it has not more than $k$ solutions. Since the number of roots of unity in $K$ is $\leq 2 d^{2}$, solutions $x$ where (9.33) is not an identity are contained in a set of cardinality $<2 d^{2} k$, as asserted in (9.27).

Comparing the leading coefficients in (9.33), it follows, since $\beta$ is not a root of unity, that there exists at most one root of unity $\zeta$ such that (9.33) holds identically in $x$. It is clear that only such a $\zeta$ can give rise to a one-parameter family $\mathcal{F}_{p^{\prime}}$ as in (9.30) having (9.29), and the lemma follows.

10. Equations $f(x) \alpha^{x}=b \beta^{y}$. In Section 9 we have treated equation (8.5) under the assumption that both $f$ and $g$ have positive degrees. 
In this section we study equation (8.5) assuming that

$$
g(y) \equiv b,
$$

i.e., that $m=0$. We assume again that we have (8.4) and that $(p, q)$ is minimal. For the sake of completeness in our applications we state

Lemma 10.1. Let $a$ and $b$ be elements of $K^{*}$. Suppose that $\alpha, \beta \in K^{*}$ are not roots of unity. Then the equation

$$
a \alpha^{x}=b \beta^{y}
$$

either has no solution $(x, y) \in \mathbb{Z}^{2}$ at all, or the set of solutions consists of the one-parameter family

$$
x=p t+p^{\prime}, \quad y=q t+q^{\prime} \quad(t \in \mathbb{Z})
$$

where $\left(p^{\prime}, q^{\prime}\right) \in \mathbb{Z}^{2}$ is the unique solution of the equation $a \alpha^{p^{\prime}}=b \beta^{q^{\prime}}$ with $0 \leq p^{\prime}<p, q^{\prime} \in \mathbb{Z}$. Moreover, if $H \geq \max \{h(\alpha), h(\beta), h(a), h(b)\}$, then

$$
p^{\prime} \leq 60 d^{6} \log H \quad \text { and } \quad\left|q^{\prime}\right| \leq 2^{11} d^{9} \log H .
$$

Proof. Only the assertion on $p^{\prime}$ and $q^{\prime}$ needs some comments. By Lemma 6.3 , we have $p^{\prime} \leq 60 d^{6} \log H$. But, moreover, $\beta^{q^{\prime}}=(a / b) \alpha^{p^{\prime}}$ implies $h(\beta)^{\left|q^{\prime}\right|} \leq h(a) h(b) h(\alpha)^{p^{\prime}}$, hence by Lemma 5.2,

$$
\left|q^{\prime}\right| \frac{1}{30 d^{3}} \leq\left(p^{\prime}+2\right) \log H
$$

and the assertion follows.

LEMMA 10.2. Let $f(x)$ and $g(x)$ be given by (8.1) and (10.1) respectively. Suppose that $f$ has at least two distinct zeros. Let $H$ be a quantity with

$$
H \geq \max \left\{2, h(a), h\left(A_{1}\right), \ldots, h\left(A_{l}\right), h(b)\right\} .
$$

Then the number of solutions $(x, y) \in \mathbb{Z}^{2}$ of equation (8.5) having

$$
|x| \geq H^{2 d(k+2)^{2}}
$$

does not exceed

$$
2^{s^{7} 2^{80 d !}} .
$$

Proof. Equation (8.5) now reads $a\left(x-A_{1}\right) \ldots\left(x-A_{l}\right)=b \beta^{y} \alpha^{-x}$. Let $D$ be a common denominator of $a, A_{1}, \ldots, A_{l}, b$. By Lemma $5.1, D$ may be chosen with

$$
D \leq H^{d(l+2)} .
$$

We have

$$
D a\left(D x-D A_{1}\right) \ldots\left(D x-D A_{l}\right)=D^{l+1} b \beta^{y} \alpha^{-x} .
$$


Notice that here $D x-D A_{i} \neq 0$ for each $i(1 \leq i \leq l)$. Now (10.8) implies

$$
\prod_{v \in S}\|D a\|_{v}\left\|D x-D A_{1}\right\|_{v} \ldots\left\|D x-D A_{l}\right\|_{v}=\prod_{v \in S}\left\|D^{l+1} b\right\|_{v} .
$$

By the definition of $D$, each factor on the left hand side of (10.9) is $\geq 1$. We suppose without loss of generality that $A_{1} \neq A_{2}$ and obtain from (10.9),

$$
\prod_{v \in S}\left\|D x-D A_{1}\right\|_{v}\left\|D x-D A_{2}\right\|_{v} \leq \prod_{v \in S}\left\|D^{l+1} b\right\|_{v} .
$$

Thus, we may conclude that

$$
\begin{array}{rl}
\prod_{v \in S} \| D & x-D A_{1}\left\|_{v}\right\| D x-D A_{2}\left\|_{v}\right\| D A_{1}-D A_{2} \|_{v} \\
\leq & \prod_{v \in S}\left\|D^{l+1} b\right\|_{v}\left\|D A_{1}-D A_{2}\right\|_{v} \\
\leq & D^{l+2} h(b) \cdot 2 h\left(A_{1}\right) h\left(A_{2}\right)<H^{d(l+3)^{2}-2} .
\end{array}
$$

On the other hand, we get

$$
\begin{aligned}
H\left(\left(D x-D A_{1}, D x-D A_{2}, D A_{1}-D A_{2}\right)\right) & \\
& =H\left(\left(x-A_{1}, x-A_{2}, A_{1}-A_{2}\right)\right) \\
& \geq \prod_{v \mid \infty}\left\|x-A_{1}\right\|_{v} \prod_{v \nmid \infty}\left\|A_{1}-A_{2}\right\|_{v} \\
& =\prod_{v \mid \infty}\left\|x-A_{1}\right\|_{v}\left\|A_{1}-A_{2}\right\|_{v}^{-1} .
\end{aligned}
$$

Let $\sigma_{1}, \ldots, \sigma_{d}$ be the isomorphic embeddings of $K$ in $\mathbb{C}$. Then (10.4) and (10.5) entail

$$
\begin{aligned}
\prod_{v \mid \infty}\left\|x-A_{1}\right\|_{v} & =\left(\prod_{i=1}^{d}\left|x-\sigma_{i} A_{1}\right|\right)^{1 / d} \geq \prod_{i=1}^{d}\left(|x|-\left|\sigma_{i} A_{1}\right|\right)^{1 / d} \\
& \geq \prod_{i=1}^{d}\left(|x|-H^{d}\right)^{1 / d}=|x|-H^{d} \geq \frac{|x|}{2} .
\end{aligned}
$$

Moreover, we have

$$
\prod_{v \mid \infty}\left\|A_{1}-A_{2}\right\|_{v} \leq 2 h\left(A_{1}\right) h\left(A_{2}\right) \leq 2 H^{2}
$$

Therefore, (10.11) implies

$$
H\left(\left(D x-D A_{1}, D x-D A_{2}, D A_{1}-D A_{2}\right)\right) \geq \frac{|x|}{4} H^{-2} .
$$


Comparing (10.12) with (10.10) we obtain using (10.5),

$$
\begin{aligned}
\prod_{v \in S} \| D x-D & A_{1}\left\|_{v}\right\| D x-D A_{2}\left\|_{v}\right\| D A_{1}-D A_{2} \|_{v} \\
& <H^{d(l+3)^{2}-2} \leq \frac{|x|^{1 / 2}}{2} H^{-1} \\
& \leq H\left(\left(D x-D A_{1}, D x-D A_{2}, D A_{1}-D A_{2}\right)\right)^{1 / 2} \\
& \leq H_{S}\left(\left(D x-D A_{1}, D x-D A_{2}, D A_{1}-D A_{2}\right)\right)^{1 / 2} .
\end{aligned}
$$

We apply Lemma 7.1 to the equation $x_{1}+x_{2}+x_{3}=0$ with

$$
x_{1}=D x-D A_{1}, \quad x_{2}=-D x+D A_{2}, \quad x_{3}=D A_{1}-D A_{2} .
$$

In view of (10.13), hypothesis (7.2) is satisfied with $\delta=1 / 2$. The conclusion is that the solutions $x_{1}, x_{2}, x_{3}$ satisfy one of

$$
2 \cdot 2^{s}(4 s d !)^{2^{37 \cdot 2 \cdot d !} s^{6} \cdot 4}
$$

linear relations, each of which may be taken as

$$
c_{1} x_{1}+c_{2} x_{2}=0,
$$

with $c_{1}, c_{2} \in K,\left(c_{1}, c_{2}\right) \neq(0,0)$. If here $c_{1}=c_{2}$, then by (10.14) we get $A_{1}=$ $A_{2}$, a contradiction. Therefore each relation (10.16) implies an equation of the shape $x+c_{3}=0$, and hence determines $x$ uniquely.

On the other hand, given $x$ there is at most one value $y$ such that $f(x) \alpha^{x}=b \beta^{y}$. Thus (10.6) follows from (10.15).

Lemma 10.3. Suppose that $f(x)=a(x-A)^{l}$ and $g(x)=b$, with $a, b, A \in$ $K, a b \neq 0$. Let $H$ be a quantity satisfying

$$
H \geq \max \{2, h(a), h(A), h(b)\} .
$$

Suppose that

$$
A \notin \mathbb{Q} .
$$

Then the number of solutions $(x, y) \in \mathbb{Z}^{2}$ of the equation

$$
f(x) \alpha^{x}=g(y) \beta^{y}
$$

(i.e., of (8.5)) with

$$
|x| \geq H^{2 d^{2}(k+2)^{2}}
$$

does not exceed

$$
2^{s^{7} 2^{80 d !}} .
$$


Proof. Let $\sigma_{1}, \ldots, \sigma_{d}$ be the isomorphic embeddings of our field $K$ in an algebraic closure. Then (10.19) implies

$$
\prod_{i=1}^{d} \sigma_{i}(a) \prod_{i=1}^{d}\left(x-\sigma_{i}(A)\right)^{l} \prod_{i=1}^{d} \sigma_{i}(\alpha)^{x}=\prod_{i=1}^{d} \sigma_{i}(b) \prod_{i=1}^{d} \sigma_{i}(\beta)^{y} .
$$

Write $\prod_{i=1}^{d} \sigma_{i}(a)=C, \sigma_{i}(A)=A_{i}(i=1, \ldots, d), \prod_{i=1}^{d} \sigma_{i}(b)=B, \prod_{i=1}^{d} \sigma_{i}(\alpha)$ $=\alpha^{\prime}, \prod_{i=1}^{d} \sigma_{i}(\beta)=\beta^{\prime}$. Then we get

$$
C \prod_{i=1}^{d}\left(x-A_{i}\right)^{l} \alpha^{\prime x}=B \beta^{\prime y} .
$$

Since by (10.18), $A \notin \mathbb{Q}$, we may suppose that $A_{1} \neq A_{2}$. So (10.22) is an equation of the type studied in Lemma 10.2.

The only problem with (10.22) is that the zeros $A_{i}$ of the polynomial on the left hand side in general will not lie in $K$ but in the normal closure of $K$. Conceivably this could have some impact in the application of Lemma 7.1. However, the proof of Lemma 7.1 (cf. [9], Section 2, and [8], Section 2) is such that in fact the result in Lemma 7.1 covers even the solutions in the normal closure of $K$ (this is the reason that (7.3) involves the parameter $d$ ! instead of simply the degree $d$ of $K$ ). Now, analyzing the proof of Lemma 10.2 we see easily that in our context everything goes through if we replace the parameter $H$ by $H^{d}$. Thus hypothesis (10.5) becomes (10.20). With this new hypothesis the conclusion remains the same, and (10.21) follows from (10.6).

We may summarize the results of this section as follows.

Lemma 10.4. Let $f(x)$ be given by (8.1) and $g(x) \equiv b$ with $b \neq 0$. Suppose that $\operatorname{deg} f>0$ and that $f$ is not of the shape $a(x-A)^{l}$ with $A \in \mathbb{Q}$. Let $H$ be a quantity with

$$
H \geq \max \left\{3, h(a), h\left(A_{1}\right), \ldots, h\left(A_{l}\right), h(b), h(\alpha)\right\} .
$$

Suppose that $\alpha \in K^{*}$ is not a root of unity. Then the number of solutions $(x, y) \in \mathbb{Z}^{2}$ of the equation

$$
f(x) \alpha^{x}=g(y) \beta^{y}
$$

with

$$
|x| \geq H^{2 d^{2}(k+2)^{2}}
$$

does not exceed

$$
2^{s^{7} 2^{80 d !}} .
$$

Moreover, solutions $(x, y) \in \mathbb{Z}^{2}$ where $(10.25)$ is violated have

$$
|y| \leq H^{27 d^{2} k^{2}} .
$$


P r o o f. It suffices to prove (10.27). We get

$$
\beta^{y}=\frac{a}{b}\left(x-A_{1}\right) \ldots\left(x-A_{l}\right) \alpha^{x},
$$

and hence by Lemma 5.1,

$$
h(\beta)^{|y|} \leq H^{2} \cdot 2^{l}|x|^{l} H^{l+|x|} .
$$

Thus Lemma 5.2 and (10.25) imply

$$
\begin{aligned}
\frac{1}{30 d^{3}}|y| & \leq(l+2+|x|) \log H+l \log |x|+l \log 2 \\
& <\left(k+2+H^{2 d^{2}(k+2)^{2}}\right) H+k \cdot 2 d^{2}(k+2)^{2} H+k H,
\end{aligned}
$$

and (10.27) follows with a simple computation.

11. Equations $f(x) \alpha^{x}=b \beta^{y}$, continued. In this section, we study the special case of equation (8.5) given by

$$
a(x-A)^{l} \alpha^{x}=b \beta^{y}
$$

where $a, b \in K^{*}, A \in \mathbb{Q}, \alpha, \beta \in K^{*}, \alpha$ and $\beta$ are not roots of unity and where $l>0$.

We suppose, moreover, that we have (8.4), i.e.,

$$
\alpha^{p}=\beta^{q} \quad(=\delta)
$$

and that here $p>0$ is minimal.

Lemma 11.1. Suppose that for all integers $u \neq 0$ we have $\alpha^{u} \notin \mathbb{Q}$. Then (11.1) has at most two solutions $(x, y) \in \mathbb{Z}^{2}$.

Proof. As $\beta$ is not a root of unity, it is clear that given $x \in \mathbb{Z}$, there is at most one $y \in \mathbb{Z}$ such that $(x, y)$ is a solution of (11.1). For a solution $(x, y)$ the relations (11.1), (11.2) imply

$$
a^{p q}(x-A)^{p q l} \delta^{q x}=b^{p q} \delta^{p y} .
$$

Write $w=p y-q x$. Then we get

$$
a^{p q}(x-A)^{p q l}=b^{p q} \delta^{w} .
$$

Now, suppose that we have two solutions $\left(x_{1}, w_{1}\right),\left(x_{2}, w_{2}\right) \in \mathbb{Z}^{2}$ of (11.3). Then

$$
\frac{\left(x_{1}-A\right)^{p q l}}{\left(x_{2}-A\right)^{p q l}}=\delta^{w_{1}-w_{2}} .
$$

Our hypothesis implies that $\delta^{u} \in \mathbb{Q}$ for $u \in \mathbb{Z}$ is only possible for $u=0$. Thus by (11.4), we have $w_{1}=w_{2}$ and consequently $\left(x_{1}-A\right)^{p q l}=\left(x_{2}-A\right)^{p q l}$. It follows that either $x_{1}=x_{2}$ or $x_{1}+x_{2}=2 A$. Thus given a solution $\left(x_{1}, w_{1}\right)$, every solution $(x, w)$ has $x=x_{1}$ or $x=2 A-x_{1}$. 
Re m ark. The hypothesis of Lemma 11.1 may be effectively verified. In fact, by Lemma 6.2, it suffices to check the powers $\alpha^{u}$ with

$$
1 \leq u \leq 2 d^{3} \text {. }
$$

In the sequel we will suppose that some nonzero integral power of $\alpha$ lies in $\mathbb{Q}$. Then naturally the same holds true for $\delta=\alpha^{p}$.

Define $u_{1}$ as the least positive integer such that

$$
\delta^{u_{1}} \in \mathbb{Q}^{p q l},
$$

and define $Q \in \mathbb{Q}$ by

$$
Q^{p q l}=\delta^{u_{1}} .
$$

Since $\delta=\alpha^{p}$, (11.7) together with (11.5), (8.6) and (6.5) implies

$$
h(Q) \leq \exp \left(120 d^{9} \log ^{2} H\right) .
$$

Lemma 11.2. Suppose that $Q \notin \mathbb{Z}$. Let $H$ be a quantity with

$$
H \geq \max \{3, h(a), h(b), h(A), h(\alpha), h(\beta)\} .
$$

Then the solutions $(x, y) \in \mathbb{Z}^{2}$ of (11.1) satisfy

$$
p y-q x<2^{22} d^{24} k \log ^{4} H .
$$

Similarly, if $Q^{-1} \notin \mathbb{Z}$, then the solutions $(x, y) \in \mathbb{Z}^{2}$ of (11.1) satisfy

$$
q x-p y<2^{22} d^{24} k \log ^{4} H \text {. }
$$

Proof. Put $p y-q x=w$; then (11.1) implies

$$
a^{p q}(x-A)^{p q l}=b^{p q} \delta^{w} .
$$

Write

$$
w=u_{1} z+u_{0}
$$

with $u_{1}$ as in (11.6), $z \in \mathbb{Z}$ and $0 \leq u_{0}<u_{1}$. We infer from (11.5) and (6.5) that

$$
u_{1} \leq 2 d^{3}|p q| l<7200 k d^{15} \log ^{2} H .
$$

Now combination of (11.6), (11.7) and (11.12) yields

$$
(b / a)^{p q} \delta^{u_{0}}=\left((x-A) Q^{-z}\right)^{p q l} \in \mathbb{Q}^{|p q l|} .
$$

If there exists $u_{0}$ in the range $0 \leq u_{0}<u_{1}$ with $(b / a)^{p q} \delta^{u_{0}} \in \mathbb{Q}^{|p q l|}$, i.e. with (11.15), then by the definition of $u_{1}$, such a $u_{0}$ is uniquely determined. (If there is no $u_{0}$ with this property, then (11.1) has no solution $(x, y) \in \mathbb{Z}^{2}$ at all.) It is clear by (11.4) that there exists an algorithm to decide whether $u_{0}$ exists and to determine $u_{0}$ effectively, if it exists.

Now suppose that $u_{0}$ is as in (11.15). Define the rational number $r$ by

$$
r^{p q l}=(b / a)^{p q} \delta^{u_{0}} .
$$


Then by (11.14) we get

$$
h(r) \leq h(b) h(a) h(\delta)^{2 d^{3}} .
$$

Combination of (11.15) and (11.16) yields

$$
x=A \pm r Q^{z} \quad \text { with } x, z \in \mathbb{Z} .
$$

Suppose that $Q \notin \mathbb{Z}$. Then for $z \in \mathbb{Z}$ with $2^{z}>h(A) h(r)$, (11.18) entails that $x \notin \mathbb{Z}$. Thus in (11.18) we have necessarily

$$
z<2(\log h(A)+\log h(r)) .
$$

Using the definition of $z$ in (11.13) together with (11.14) and (11.17) we obtain

$$
\begin{aligned}
w=u_{1} z+u_{0} & <2 d^{3}|p q| l(2(\log h(A)+\log h(r))+1) \\
& \leq 2^{22} d^{24} k \log ^{4} H
\end{aligned}
$$

and this is (11.10).

(11.11) follows in the same way.

Lemma 11.3. Suppose that $Q \notin \mathbb{Z}$. Let $H$ be as in (11.9). Then the solutions $(x, y) \in \mathbb{Z}^{2}$ of (11.1) with

$$
p y-q x \geq 0
$$

satisfy

$$
|x| \leq \exp \left(2^{13} d^{18} \log ^{4} H\right), \quad|y| \leq \exp \left(2^{14} d^{18} \log ^{4} H\right) .
$$

Similarly, if $Q^{-1} \notin \mathbb{Z}$, then the solutions $(x, y) \in \mathbb{Z}^{2}$ of (11.1) with

$$
p y-q x \leq 0
$$

satisfy (11.21).

Proof. We have (11.3), i.e.,

$$
a^{p q}(x-A)^{p q l}=b^{p q} \delta^{p y-q x} .
$$

So if (11.20) holds true, we have

$$
h(x-A) \leq\left(h(b) h(a) h(\delta)^{(p y-q x) /|p q|}\right)^{1 / l}
$$

and therefore since $p y-q x=w$ and by (11.19),

$$
\begin{aligned}
|x| & \leq\left(h(b) h(a) h(\delta)^{(p y-q x) /|p q|}\right)^{1 / l}+h(A) \\
& \leq H^{2} \log h(\delta) \exp \left(4 d^{2}(\log h(A)+\log h(r))\right)+h(A) .
\end{aligned}
$$

Recall that $\delta=\alpha^{p}$, so that by $(6.5), \log h(\delta) \leq 60 d^{6} \log ^{2} H$, and from (11.17) we get

$$
\log h(r) \leq 2 \log H+120 d^{9} \log ^{2} H<2^{7} d^{9} \log ^{2} H,
$$


which finally implies that $|x| \leq \exp \left(2^{13} d^{18} \log ^{4} H\right)$. But then (11.10) and (11.20) in conjunction with (9.15) give $|y| \leq \exp \left(2^{14} d^{18} \log ^{4} H\right)$, and so (11.21) follows.

The combination (11.22), (11.21) may be shown in the same way.

To finish our study of equation (11.1), we still have to deal with the cases when $Q$ in (11.7) satisfies

$$
Q \in \mathbb{Z} \quad \text { or } \quad Q^{-1} \in \mathbb{Z} .
$$

It turns out that this is the most complicated case. In [11] we called an equation

$$
f(x) \alpha^{x}=g(y) \beta^{y}
$$

exceptional of type 1 if $f$ has exactly one root $A$ (of arbitrary multiplicity) and if this root lies in $\mathbb{Q}$, if $g$ is a nonzero constant and $\alpha, \beta \in K^{*}$ are not roots of unity but such that there exist nonzero integers $p, q$ with $\alpha^{p}=\beta^{q}=$ $\delta$, and such that moreover some power $\delta^{u}$ with $u \in \mathbb{Z} \backslash\{0\}$ lies in $\mathbb{Z}$. We called the equation exceptional of type 2 if the roles of $f$ and $g$ are interchanged.

So let us now deal with the exceptional equation of type 1. Suppose $Q \in \mathbb{Z}$. Equation (11.1) gives (11.18), i.e.,

$$
x=A \pm r Q^{z},
$$

and we ask for solutions $(x, z) \in \mathbb{Z}^{2}$. Put

$$
A=B / D, \quad r=C / D \quad \text { with } D>0
$$

and with $B, C, D \in \mathbb{Z},(B, C, D)=1$. Then $D \leq h(A) h(r)$ and thus by (11.23) and (6.5),

$$
D \leq \exp \left(2^{8} d^{9} \log ^{2} H\right) .
$$

Then $x$ given by (11.25) lies in $\mathbb{Z}$ if

$$
B \pm C Q^{z} \equiv 0(\bmod D) .
$$

Put $D=D_{1} D_{2}$, where $D_{1}$ is made up of primes dividing $Q$ and $D_{2}$ of other primes. Then for $z$ with $2^{z}>D_{1}$ we get $Q^{z} \equiv 0\left(\bmod D_{1}\right)$, and in view of (11.28) this entails

Lemma 11.4. Suppose that $Q \in \mathbb{Z}$ and that $B \not \equiv 0\left(\bmod D_{1}\right)$. Then the solutions $(x, y) \in \mathbb{Z}^{2}$ of (11.1) satisfy

$$
|x| \leq \exp \left(2^{17} d^{18} \log ^{4} H\right), \quad|y| \leq \exp \left(2^{18} d^{18} \log ^{4} H\right) .
$$

Proof. By Lemma 11.3 we may suppose that $p y-q x>0$. Recall the definition of $z$ in (11.13). We have $u_{1} z+u_{0}=p y-q x$. Our hypothesis implies that only such $z$ are admitted for which $2^{z} \leq D_{1}$. We infer from (11.27) that 
necessarily $z \leq 2^{9} d^{9} \log ^{2} H$. Combining this with (11.14), we get

$$
p y-q x \leq 2^{10} d^{12}|p q| l \log ^{2} H .
$$

The proof may now be finished in exactly the same way as in Lemma 11.3.

It is clear that we get a result analogous to Lemma 11.4 if $Q^{-1} \in \mathbb{Z}$.

We now suppose that $B$ in $(11.28)$ has $B \equiv 0\left(\bmod D_{1}\right)$. We detail only the case when in (11.28) we have the + sign. By Lemma 11.3, it suffices to find the solutions $z \in \mathbb{Z}, z \geq 0$, of the congruence

$$
B+C Q^{z} \equiv 0(\bmod D),
$$

since for $z<0$ we have $p y-q x \leq 0$ and that case is treated in (11.22), (11.21). Values $z$ with $2^{z} \leq D_{1}$ lead to solutions $x, y$ of (11.1) with (11.29) (this follows at once from the proof of Lemma 11.4).

If (11.30) has a solution $z$ with $2^{z}>D_{1}$ at all, then it has such a solution $z^{+}$in the range

$$
\frac{\log D_{1}}{\log 2}<z^{+} \leq \frac{\log D_{1}}{\log 2}+D_{2} .
$$

Let $z^{+}$be minimal with (11.31) such that (11.30) is satisfied for $z=z^{+}$. By (11.27), $z^{+}$is effectively computable. By definition of $D_{2}$, we have $\left(Q, D_{2}\right)=$ 1. Let $v>0$ be the least solution of $Q^{v} \equiv 1\left(\bmod D_{2}\right)$. Again by (11.27) we have

$$
0<v \leq \varphi\left(D_{2}\right) \leq \exp \left(2^{8} d^{9} \log ^{2} H\right) .
$$

It is now clear that the solutions $z$ of (11.30) with $z>\left(\log D_{1}\right) / \log 2$ are given by

$$
z=z^{+}+v s \quad(s \in \mathbb{Z}, s \geq 0) .
$$

In view of (11.18) we therefore obtain

$$
x=r Q^{z^{+}}\left(Q^{v}\right)^{s}+A=E^{\prime} R_{0}^{s}+A \quad(s \in \mathbb{Z}, s \geq 0),
$$

where $E^{\prime}=r Q^{z^{+}}$and $R_{0}=Q^{v}$. Now by (11.3) we get

$$
\begin{aligned}
p y & =w+q x=u_{1} z+u_{0}+q x=q E^{\prime} R_{0}^{s}+u_{1} v s+u_{1} z^{+}+u_{0}+q A \\
& =q E^{\prime} R_{0}^{s}+F^{\prime} s+G^{\prime},
\end{aligned}
$$

say, with $F^{\prime}=u_{1} v$ and $G^{\prime}=u_{1} z^{+}+u_{0}+q A$. But $y$ needs to be an integer. So the question is: for which $s$ do we have

$$
q E^{\prime} R_{0}^{s}+F^{\prime} s+G^{\prime} \equiv 0(\bmod p) ?
$$

Write $p=p_{1} p_{2}$ where $p_{1}$ is a product of primes dividing $R_{0}$, and $p_{2}$ is a product of other primes. If $s$ is so large that $2^{s}>h(r) p$, then the rational number $q E^{\prime} R_{0}^{s}$ will have a numerator $\equiv 0\left(\bmod p_{1}\right)$. In view of $(11.23)$ 
and (6.5) this will be satisfied if

$$
s>2^{8} d^{9} \log ^{2} H \text {. }
$$

Solutions where (11.36) is violated lead to $z=z^{+}+v s \leq \exp \left(2^{9} d^{9} \log ^{2} H\right)$ by (11.27), (11.32) and (11.37), and this implies an effective bound for the corresponding solutions $x$ and $y$ of (11.1) (cf. the proof of Lemma 11.3). So it suffices to solve (11.36) for $s \geq s_{0}$ with $s_{0}=\left[2^{8} d^{9} \log ^{2} H+1\right]$, and consequently we have to solve simultaneously

$$
F^{\prime} s+F^{\prime} s_{0}+G^{\prime} \equiv 0\left(\bmod p_{1}\right)
$$

and

$$
q E^{\prime} R_{0}^{s_{0}} R_{0}^{s}+F^{\prime} s+F^{\prime} s_{0}+G^{\prime} \equiv 0\left(\bmod p_{2}\right)
$$

with $s \in \mathbb{Z}, s \geq 0$.

If the congruence (11.38) is solvable at all, then the solutions $s$ run through certain residue classes $\bmod p_{1}$, and since we have effective bounds for all parameters in (11.38), we may compute these classes effectively. Now, given such a residue class $s=p_{1} t+p_{1}^{\prime}(t \in \mathbb{Z}, t \geq 0)$, we have to solve (11.39) for $s$ in this class, i.e.,

$$
\begin{aligned}
q E^{\prime} R_{0}^{s_{0}+p_{1}^{\prime}}\left(R_{0}^{p_{1}}\right)^{t}+F^{\prime} p_{1} t+F^{\prime}\left(s_{0}+p_{1}^{\prime}\right)+G^{\prime} \equiv & 0\left(\bmod p_{2}\right) \\
& (t \in \mathbb{Z}, t \geq 0) .
\end{aligned}
$$

Since $\left(R_{0}, p_{2}\right)=1$, the residue class of $\left(R_{0}^{p_{1}}\right)^{t}\left(\bmod p_{2}\right)$ depends only upon the residue class of $t \bmod \varphi\left(p_{2}\right)$ and the solutions $t$ of (11.40) lie in suitable residue classes $\bmod p_{2} \varphi\left(p_{2}\right)$ (if there are solutions $t$ at all). Again since we have effective bounds for all the parameters in (11.40), it is decidable whether (11.40) admits solutions, and in the affirmative case we may determine effectively all residue classes.

Collecting our results, we see that the solutions $s$ of (11.36) consist of certain residue classes $\bmod p_{1} p_{2} \varphi\left(p_{2}\right)$ and these classes are effectively computable. Let $P t+P^{\prime}(t \in \mathbb{Z}, t \geq 0)$ be such a residue class. Then by (11.34) we get $x(t)=r Q^{z^{+}} Q^{v P^{\prime}}\left(Q^{v P}\right)^{t}+A$, and so

$$
x(t)=E p R^{t}+A \quad(t \in \mathbb{Z}, t \geq 0),
$$

where

$$
E=r Q^{z^{+}+v P^{\prime}} p^{-1}, \quad R=Q^{v P} .
$$

Moreover, we obtain

$$
\begin{aligned}
p y & =q x+w=q x+u_{1} z+u_{0}=q x+u_{1} v s+u_{1} z^{+}+u_{0} \\
& =q x+u_{1} v P t+u_{1} v P^{\prime}+u_{1} z^{+}+u_{0},
\end{aligned}
$$

and hence by (11.41),

$$
y(t)=E q R^{t}+F t+G \quad(t \in \mathbb{Z}, t \geq 0),
$$


where

$$
F=u_{1} v P, \quad G=q A+u_{1} v P^{\prime}+u_{1} z^{+}+u_{0} .
$$

All this was when $Q \in \mathbb{Z}$ and we have the $+\operatorname{sign}$ in (11.28). If we have the sign in (11.28), then defining $z^{-}$with respect to the congruence $B-C Q^{z} \equiv 0$ $(\bmod D)$ in the same way as we got $z^{+}$, all the above still holds true mutatis mutandis.

If, however, $Q^{-1} \in \mathbb{Z}$, then in the above construction we have to study solutions $x, y$ with $0>w=p y-q x=u_{1} z+u_{0}$, and we have to focus on $z$ with $z<0$. Again we obtain exponential families $x(t), y(t)$ of the type (11.41), (11.43). We only have to replace $z^{+}$by $-z^{+}$or $z^{-}$by $-z^{-}$respectively, and $v$ by $-v$. We remark that in (11.41) and (11.43), the quantities $R$ and $F$ do not depend upon the particular residue class $\bmod P$ and that, moreover, we have $F \neq 0$.

We summarize what we have shown.

Lemma 11.5. Suppose that equation (11.1) is exceptional of type 1. Then apart from finitely many solutions $x, y$ which may be effectively computed, the solutions $(x, y)$ of (11.1) make up a finite number (possibly zero) of oneparameter exponential families, each of type (11.41), (11.43). There exists an algorithm to decide whether such exponential families do occur at all. If such families do occur, then they are effectively computable.

12. Summary on equations $f(x) \alpha^{x}=g(y) \beta^{y}$. We collect the results on equation (8.5) proved in the preceding sections. We assume throughout that (8.1)-(8.6) are valid.

Proposition 12.1. Suppose that (8.5) is not exceptional. Then there exists an algorithm to decide whether (8.5) has infinitely or only finitely many solutions.

(a) If (8.5) has only finitely many solutions, then all but at most

$$
M=2^{s^{7} 2^{45 d !(2 k) !}}
$$

solutions have

$$
\max (|x|,|y|) \leq c(d, k, H)
$$

with effectively computable $c(d, k, H)$.

(b) If (8.5) has infinitely many solutions, then there is a unique linear one-parameter family

$$
x(t)=p t+p^{\prime}, \quad y(t)=q t+q^{\prime} \quad(t \in \mathbb{Z})
$$

with $0 \leq p^{\prime}<p$, such that all but at most $M$ solutions lie in this family or have (12.2). The family (12.3) and the bound $c(d, k, H)$ are effectively computable. 
Proof. The case when both $f$ and $g$ have positive degree follows from Lemmata 9.2 and 9.3. The bound in (12.1) is the sum of the bounds in (9.8) and $k^{2}$ times the bound in (9.27).

Lemma 10.1 covers the case when both $f$ and $g$ are constants. The remaining cases are treated in Lemmata 10.4, 11.1 and 11.3.

We remark that the bound $2^{s^{7} 2^{80 d !}}$ in Lemma 10.4 is smaller than the bound in (12.1) since $k \geq 1$.

Proposition 12.2. Suppose that (8.5) is exceptional of type 1. Then there exists an algorithm to decide whether (8.5) has infinitely many or only finitely many solutions. Moreover:

(a) If (8.5) has only finitely many solutions, then each solution satisfies

$$
\max (|x|,|y|) \leq c(d, k, H) .
$$

(b) If (8.5) has infinitely many solutions, then they make up finitely many one-parameter exponential families, each of the type

$$
x_{j}(t)=p E_{j} R^{t}+A, \quad y_{j}(t)=q E_{j} R^{t}+F t+G_{j} \quad(t \in \mathbb{Z}, t \geq 0)
$$

$(1 \leq j \leq c(d, k, H))$, and possibly some further solutions with (12.4).

Here the families (12.5) are effectively computable. Moreover, $E_{j}, G_{j}$ are rational numbers, $F$ is a nonzero integer that does not depend upon the particular family, and $R=\alpha^{u} \in \mathbb{Z}$ for some $u \in \mathbb{Z} \backslash\{0\}$. The constant in (12.4) is effective.

Proof. The assertion follows at once from Lemmata 11.3 and 11.4 and from formulas (11.41)-(11.44) in the proof of Lemma 11.4.

13. Intersections of solution families. We consider a system of equations

$$
\begin{aligned}
& f_{1}(x) \alpha_{1}^{x}=g_{1}(y) \beta_{1}^{y}, \\
& f_{2}(x) \alpha_{2}^{x}=g_{2}(y) \beta_{2}^{y},
\end{aligned} \quad(x, y) \in \mathbb{Z}^{2} .
$$

Here we suppose that $f_{1}, f_{2}, g_{1}, g_{2}$ are nonzero polynomials as in (8.1), (8.2), of respective degrees $l_{1}, l_{2}, m_{1}, m_{2}$, with

$$
\max \left\{l_{1}, l_{2}, m_{1}, m_{2}\right\}<k .
$$

We assume that $\alpha_{1}, \alpha_{2}, \beta_{1}, \beta_{2} \in K^{*}$, that $\alpha_{2}$ and $\beta_{2}$ are not roots of unity and that

$$
\alpha_{i}^{p}=\beta_{i}^{q} \quad \text { for } i=1,2,
$$

where $p$ and $q$ are nonzero integers such that $p>0$ is minimal with a relation (13.4). Our hypotheses are such that at least equation (13.2) is of the shape 
studied in Sections 8-12. In studying simultaneously (13.1) and (13.2) we distinguish the following cases.

(i) $\alpha_{1}$ and $\beta_{1}$ are not roots of unity;

(ii) $\alpha_{1}$ and $\beta_{1}$ are roots of unity.

We first treat alternative (i).

We will make throughout the hypothesis

(H) Both (13.1) and (13.2) admit infinitely many solutions.

In view of Propositions 12.1 and 12.2 this will be no restriction.

If we choose $\left(p_{1}, q_{1}\right)$ and $\left(p_{2}, q_{2}\right)$ in $\mathbb{Z}^{2}$ minimal such that $\alpha_{i}^{p_{i}}=\beta_{i}^{q_{i}}$, then the pair $(p, q)$ in (13.4) will be such that $p$ is the least common multiple of $p_{1}$ and $p_{2}$.

LEMMA 13.1. Suppose that neither (13.1) nor (13.2) is exceptional and that we have $(\mathrm{H})$. Then the intersection of the linear families

$$
x_{1}\left(t_{1}\right)=p_{1} t_{1}+p_{1}^{\prime}, \quad y_{1}\left(t_{1}\right)=q_{1} t_{1}+q_{1}^{\prime} \quad\left(t_{1} \in \mathbb{Z}\right)
$$

and

$$
x_{2}\left(t_{2}\right)=p_{2} t_{2}+p_{2}^{\prime}, \quad y_{2}\left(t_{2}\right)=q_{2} t_{2}+q_{2}^{\prime} \quad\left(t_{2} \in \mathbb{Z}\right)
$$

from (12.3) either is empty or equals

$$
x(t)=p t+p^{\prime}, \quad y(t)=q t+q^{\prime} \quad(t \in \mathbb{Z}),
$$

where $p$ is the least common multiple of $p_{1}$ and $p_{2}$ and where $0 \leq p^{\prime}<p$ is uniquely determined.

Proof. Suppose that the intersection of the families (13.5) and (13.6) is not empty. Let $t_{1}^{(0)}, t_{2}^{(0)} \in \mathbb{Z}$ be parameters having

$$
p_{1} t_{1}^{(0)}+p_{1}^{\prime}=p_{2} t_{2}^{(0)}+p_{2}^{\prime}, \quad q_{1} t_{1}^{(0)}+q_{1}^{\prime}=q_{2} t_{2}^{(0)}+q_{2}^{\prime} .
$$

Then the intersection consists of points

$$
x\left(t_{1}\right)=p_{1} t_{1}+p_{1} t_{1}^{(0)}+p_{1}^{\prime}, \quad y\left(t_{1}\right)=q_{1} t_{1}+q_{1} t_{1}^{(0)}+q_{1}^{\prime} \quad\left(t_{1} \in \mathbb{Z}\right),
$$

where $t_{1}$ satisfies the equation $p_{1} t_{1}=p_{2} t_{2}$ with $t_{1}, t_{2} \in \mathbb{Z}$. Here $t_{1}$ may be written as $t_{1}=p_{2} t /\left(p_{1}, p_{2}\right)$ with $t \in \mathbb{Z}$ and the assertion follows.

Lemma 13.2. Suppose that one of (13.1), (13.2) is exceptional and the other is not. Suppose, moreover, that we have hypothesis $(\mathrm{H})$. Then the intersection of the linear family (12.3) with the union of the exponential families (12.5) contains at most two points $(x, y)$.

P r o of. Suppose without loss of generality that the linear family is

$$
x_{1}\left(t_{1}\right)=p_{1} t_{1}+p_{1}^{\prime}, \quad y_{1}\left(t_{1}\right)=q_{1} t_{1}+q_{1}^{\prime} .
$$

Then we get $p y_{1}\left(t_{1}\right)-q x_{1}\left(t_{1}\right)=p q_{1}^{\prime}-q p_{1}^{\prime}$. As for the exponential families, let us go back in the proof of Lemma 11.5. 
The exponential families of the lemma are contained in the family

$$
x(s)=E^{\prime} R_{0}^{s}+A, \quad y(s)=\frac{p}{q} E^{\prime} R_{0}^{s}+\frac{F^{\prime}}{p} s+G^{\prime} \quad(s \in \mathbb{Z}, s \geq 0)
$$

in (11.34) and (11.35), and possibly a second family of the same type corresponding to the - sign in (11.28). (Recall that in (13.7), $y(s)$ is not necessarily an integer.)

Now, $F^{\prime} \neq 0$ in (13.7) and we get $p y(s)-q x(s)=F^{\prime} s+p G^{\prime}-q A^{\prime}$, and the intersection with the linear family leads to parameters $s$ with $F^{\prime} s+$ $p G^{\prime}-q A^{\prime}=p q_{1}^{\prime}-q p_{1}^{\prime}$. Hence we get at most one such parameter $s$. As we have to consider at most two families of type (13.7), the assertion follows.

LEMmA 13.3. Suppose that one of (13.1), (13.2) is exceptional of type 1 and the other is exceptional of type 2. Suppose that hypothesis $(\mathrm{H})$ holds true. Let $L_{1}$ be the union of the exponential families (12.5) coming from (13.1), and similarly $L_{2}$ from (13.2). Then

$$
\left|L_{1} \cap L_{2}\right| \leq 2^{2^{52} s^{7}} .
$$

Proof. We suppose without loss of generality that (13.1) is of type 1 and (13.2) of type 2. As in the proof of Lemma 13.2, we go back to (11.34), (11.35).

Therefore $L_{1}$ is contained in the union of two exponential families $x_{1}\left(s_{1}\right)$, $y_{1}\left(s_{1}\right)$ where

$$
x_{1}\left(s_{1}\right)=E_{1} R_{1}^{s_{1}}+A_{1} \quad\left(s_{1} \in \mathbb{Z}\right)
$$

with $E_{1} \in \mathbb{Q}^{*}, A_{1} \in \mathbb{Q}$ and $R_{1} \in \mathbb{Z},\left|R_{1}\right|>1$. Similarly $L_{2}$ is contained in the union of two exponential families $x_{2}\left(s_{2}\right), y_{2}\left(s_{2}\right)$, where

$$
x_{2}\left(s_{2}\right)=E_{2} R_{2}^{s_{2}}+F s+G
$$

with $E_{2} \in \mathbb{Q}^{*}, R_{2} \in \mathbb{Z},\left|R_{2}\right|>1, F \in \mathbb{Q}^{*}, G \in \mathbb{Q}$. Moreover, as $R_{1}$ is an integral power of $\alpha_{1}$ and $R_{2}$ an integral power of $\alpha_{2}, R_{1}$ and $R_{2}$ are both $S$-units. The equation $x_{1}\left(s_{1}\right)=x_{2}\left(s_{2}\right)$ leads to an equation

$$
E_{1} R_{1}^{s_{1}}=E_{2} R_{2}^{s_{2}}+\left(F s_{2}+C\right) \cdot 1^{s_{2}},
$$

and hence is of the type covered by Theorem 1 (notice that the left hand side and the right hand side in (13.9) are not related). The conclusion is that (13.9) has not more than

$$
2^{2^{51} s^{7}}
$$

solutions $\left(s_{1}, s_{2}\right) \in \mathbb{Z}^{2}$. Allowing a factor 4 for the two alternatives we possibly have in $L_{1}$ as well as in $L_{2}$, we finally get the bound $2^{2^{52} s^{7}}$, as asserted. 
Lemma 13.4. Suppose that both (13.1) and (13.2) are exceptional of type 1. Suppose, moreover, that $\left|\alpha_{1}\right|>1$ and $\left|\alpha_{2}\right|<1$. Assume that hypothesis $(\mathrm{H})$ holds true. Then the intersection of the set of exponential families $L_{1}$ corresponding to (13.1) and the set of exponential families $L_{2}$ corresponding to (13.2) is empty.

P r o o f. Let $\left(x_{1}\left(s_{1}\right), y_{1}\left(s_{1}\right)\right)$ be an exponential family belonging to $\alpha_{1}$ and $\left(x_{2}\left(s_{2}\right), y_{2}\left(s_{2}\right)\right)$ be an exponential family belonging to $\alpha_{2}$. The construction of the families in Section 11 is such that (cf. Lemma 11.3) for $s_{i} \in \mathbb{Z}, s_{i} \geq 0$, we have $p y_{1}\left(s_{1}\right)-q x_{1}\left(s_{1}\right)>0$ and $p y_{2}\left(s_{2}\right)-q x_{2}\left(s_{2}\right)<0$. The assertion follows.

Lemma 13.5. Suppose that both (13.1) and (13.2) are exceptional of type 1. Suppose, moreover, that $\left|\alpha_{1}\right|>1$ and $\left|\alpha_{2}\right|>1$. Assume that hypothesis $(\mathrm{H})$ holds true. Let $L_{1}$ be the union of the exponential families from (13.1) and $L_{2}$ the union of the exponential families from (13.2). Then it is decidable whether $L_{1} \cap L_{2}$ is infinite or not. $L_{1} \cap L_{2}$ is infinite if and only if $f_{1}$ and $f_{2}$ have the same rational zero and $\alpha_{1}^{u_{1}}=\alpha_{2}^{u_{2}}$ for some nonzero integers $u_{1}, u_{2}$. Moreover:

(a) If $L_{1} \cap L_{2}$ is finite, then it is of cardinality at most

$$
M:=2^{2^{1080} s^{7}} .
$$

(b) If $L_{1} \cap L_{2}$ is infinite, then it consists of exponential families $x_{j}(t)$, $y_{j}(t)(t \in \mathbb{Z}, t \geq 0)(1 \leq j \leq c(d, k, H))$ of the type (12.5), plus at most $M$ further elements. These families are effectively computable. Here the parameter $R$ of the families is an integral power of both $\alpha_{1}$ and $\alpha_{2}$.

Proof. To get in (13.10) a bound $M$ that is independent of heights, it is suitable not to use directly the different exponential families we have in Proposition 12.2, but to go back to the proof of Lemma 11.5, as we have already done in proving Lemmata 13.2 and 13.3. We got our exponential families (12.5) by intersecting (11.34) and (11.35), i.e.,

$$
x(s)=E^{\prime} R_{0}^{s}+A, \quad y(s)=\frac{q}{p} E^{\prime} R_{0}^{s}+\frac{F^{\prime}}{p} s+\frac{G^{\prime}}{p} \quad(s \in \mathbb{Z}, s \geq 0)
$$

with $\mathbb{Z}^{2}$. (There may be two such families, if in (11.25) the - sign occurs as well.)

To keep $L_{1} \cap L_{2}$ under control, we first study the intersection of two families (13.11) (never mind whether the points are in $\mathbb{Z}^{2}$ or not) and only when we have this intersection under control, will we ask for points in the intersection whose components are integers. 
We have to solve a system

$$
x_{1}\left(s_{1}\right)=x_{2}\left(s_{2}\right), \quad y_{1}\left(s_{1}\right)=y_{2}\left(s_{2}\right)
$$

where $x_{i}\left(s_{i}\right), y_{i}\left(s_{i}\right)$ is of the type (13.11). The first equation in (13.12) yields a relation

$$
E^{(1)} R_{0}^{(1) s_{1}}+A^{(1)}=E^{(2)} R_{0}^{(2) s_{2}}+A^{(2)},
$$

say. We may apply Theorems 1 and 2 and conclude that apart from possible solutions in a set of cardinality $\leq 2^{2^{1080} s^{7}}$, this implies

$$
\begin{aligned}
E^{(1)} R_{0}^{(1) s_{1}}=E^{(2)} R_{0}^{(2) s_{2}}, \quad A^{(1)}= & A^{(2)}, \\
& \text { where } s_{1}, s_{2} \in \mathbb{Z}, s_{1}, s_{2} \geq 0 .
\end{aligned}
$$

We recall from (11.1) and (11.34) that $A^{(1)}, A^{(2)}$ are the zeros of $f_{1}$ and $f_{2}$ respectively. As $R^{(1)}$ and $R^{(2)}$ are integral powers of $\alpha_{1}, \alpha_{2}$ respectively, $R^{(1)}$ and $R^{(2)}$ are bounded in terms of $d$ and $H$. So, either we have a relation

$$
R^{(1) w_{1}}=R^{(2) w_{2}}
$$

with $w_{1}, w_{2} \in \mathbb{N}$ and bounded in terms of $d$ and $H$, or (13.13) has not more than one solution. (The bound in (13.10) is so generous that it easily takes care also of such a single additional solution.) If (13.14) is solvable, then the solutions $\left(s_{1}, s_{2}\right)$ of $(13.13)$ are of the shape

$$
s_{1}=w_{1} t+s_{1}^{(0)}, \quad s_{2}=w_{2} t+s_{2}^{(0)} \quad(t \in \mathbb{Z}, t \geq 0),
$$

where $w_{1}, w_{2}$ is a minimal solution of (13.14) and where $s_{1}^{(0)}, s_{2}^{(0)}$ is a minimal solution of (13.13). It is clear that $w_{1}, w_{2}, s_{1}^{(0)}, s_{2}^{(0)}$ are computable. We next have to check the second equation in (13.12). In view of (13.13), and with our values of $s_{1}, s_{2}$, this reduces to

$$
F^{(1)}\left(w_{1} t+s_{1}^{(0)}\right)+G^{(1)}=F^{(2)}\left(w_{2} t+s_{2}^{(0)}\right)+G^{(2)} \quad(t \in \mathbb{Z}, t \geq 0),
$$

and hence it either has at most one solution $t$ or it is an identity in $t$.

In the latter case, we have to apply the considerations of Section 11 to the family

$$
\begin{aligned}
& x(t)=E^{(1)} R_{0}^{(1) s_{1}^{(0)}}\left(R_{0}^{(1) w_{1}}\right)^{t}+A^{(1)}, \\
& y(t)=\frac{q}{p} E^{(1)} R_{0}^{(1) s_{1}^{(0)}}\left(R_{0}^{(1) w_{1}}\right)^{t}+\frac{F^{(1)}}{p} w_{1} t+\frac{F^{(1)} s_{1}^{(0)}+G^{(1)}}{p} \\
& (t \in \mathbb{Z}, t \geq 0)
\end{aligned}
$$

to guarantee that we get only values $y(t) \in \mathbb{Z}$. So we follow the considerations after $(11.36)$ with $E^{\prime}$ replaced by $E^{(1)} R_{0}^{(1) s_{1}^{(0)}}, R_{0}$ replaced by $R_{0}^{(1) w_{1}}, F^{\prime}$ 
replaced by $F^{(1)} w_{1}$, and $G^{\prime}$ replaced by $F^{(1)} s_{1}^{(0)}+G^{(1)}$. Again we see that either we get no solutions $t$ at all, or we get solutions $t$ that make up certain residue classes. The details may be left to the reader.

Since $R^{(1)}$ is an integral power of $\alpha_{1}$ and $R^{(2)}$ is an integral power of $\alpha_{2}$, it also follows from (13.14) that the parameter $R_{0}=R_{0}^{(1) w_{1}}$ occurring in the final families we get is an integral power of both $\alpha_{1}$ and $\alpha_{2}$.

We now treat alternative (ii), where $\alpha_{1}$ and $\beta_{1}$ are roots of unity.

Lemma 13.6. Suppose that

$$
\max \left\{\operatorname{deg} f_{1}, \operatorname{deg} g_{1}\right\}>0 .
$$

Suppose, moreover, that (13.2) is exceptional and admits a set $L_{2}$ of exponential families (12.5) among its solutions. Then the intersection of $L_{2}$ with the set of solutions $(x, y)$ of (13.1) has cardinality not exceeding

$$
4 k^{2} \text {. }
$$

Proof. $L_{2}$ is contained in two families $x_{i}(s), y_{i}(s), i=1,2$, each of the shape (11.34), (11.35), i.e.,

$$
x(s)=E R^{s}+A, \quad y(s)=\frac{q}{p} E R^{s}+F s+G \quad(s \in \mathbb{Z})
$$

with $|R|>1, R \in \mathbb{Z}, F \neq 0, E, A, F, G \in \mathbb{Q}$. Since $\alpha_{1}$ and $\beta_{1}$ are roots of unity, (13.1) implies

$$
f_{1}(x(s)) \bar{f}_{1}(x(s))=g_{1}(y(s)) \bar{g}_{1}(y(s)) .
$$

Combination of (13.18) and (13.19) gives an equation of the type

$$
P_{0}(s) R^{(2 k-2) s}+P_{1}(s) R^{(2 k-3) s}+\ldots+P_{2 k-3}(s) R^{s}+P_{2 k-2}(s)=0
$$

where the $P_{i}$ in (13.20) are polynomials with real coefficients and of degree $\leq i(0 \leq i \leq 2 k-2)$. Since $F \neq 0$ in (13.18), and by (13.16), we may conclude that not all polynomials $P_{i}$ in (13.20) are identically zero.

But then an easy application of Rolle's Theorem (cf. Pólya-Szegö [6], p. 48, Aufgabe 75) shows that (13.20) has less than $2 k^{2}$ solutions $s \in \mathbb{R}$. Allowing a factor two for the possible second family $x_{2}(s), y_{2}(s)$ making up $L_{2}$, we get the assertion.

Lemma 13.7. Suppose that in (13.1) we have $f_{1}(x)=a, g_{1}(x)=b$. Suppose, moreover, that (13.2) is exceptional and admits a set $L_{2}$ of exponential families of type (12.5) among its solutions. Then the intersection of $L_{2}$ with the set of solutions $(x, y)$ of (13.1) consists of a finite set which is effectively computable and possibly a finite set of exponential families again of type (12.5), and these families are effectively computable.

Proof. Let $w$ be the smallest natural number such that

$$
\alpha_{1}^{w}=\beta_{1}^{w} .
$$


By Lemma 6.1, $w \leq 2 d^{2}$. Write $x=w x^{\prime}+w_{x}$ and $y=w y^{\prime}+w_{y}$ with $0 \leq w_{x}<w$ and $0 \leq w_{y}<w$. Thus the problem consists in determining the intersections of exponential families $x(s), y(s)$ with residue classes $x \equiv w_{x}$ $(\bmod w), y \equiv w_{y}(\bmod w)$. As was shown in Section 11, this is effectively solvable and leads to a finite set and possibly some exponential families. (Since $w \leq 2 d^{2}$, everything is under control.)

LEMMA 13.8. Suppose that (13.2) is not exceptional, but has a linear one-parameter family (12.3) among its solutions. Then the intersection of this family with the set of solutions of (13.1) consists of a set of cardinality not exceeding

$$
2 d^{2} k
$$

and possibly a (unique) linear family

$$
x(t)=p t+p^{\prime}, \quad y(t)=q t+q^{\prime} \quad(t \in \mathbb{Z}),
$$

where $0 \leq p^{\prime}<p$.

Proof. Let $\left(p_{2}, q_{2}\right)$ be a minimal pair with $\alpha_{2}^{p_{2}}=\beta_{2}^{q_{2}}$ and $p_{2}>0$. Then (13.2) gives the unique linear family

$$
x(t)=p_{2} t+p_{2}^{\prime}, \quad y(t)=q_{2} t+q_{2}^{\prime} \quad(t \in \mathbb{Z})
$$

with $0 \leq p_{2}^{\prime}<p_{2}$. Pick $w \in \mathbb{N}$ minimal with $\alpha_{1}^{w}=\beta_{1}^{w}$. By Lemma 6.1, we have $w \leq 2 d^{2}$. Therefore, the pair $p, q$ in (13.4) has $p=v p_{2}, q=v q_{2}$ with $v \in \mathbb{N}, v \leq 2 d^{2}$.

The family (13.24) splits into $v$ families

$$
x(t)=p t+p^{\prime}, \quad y=q t+q^{\prime} \quad(t \in \mathbb{Z})
$$

with $0 \leq p^{\prime}<p$ (in fact, $p^{\prime}=p_{2}^{\prime}+i p_{2}$ and $q^{\prime}=q_{2}^{\prime}+i q_{2}$ for some $i$ with $0 \leq i<v)$.

Now, given a family (13.25), equation (13.1) becomes

$$
f_{1}\left(p t+p^{\prime}\right) \alpha_{1}^{p^{\prime}}=g_{1}\left(q t+q^{\prime}\right) \beta_{1}^{q^{\prime}} .
$$

This is a polynomial equation in $t$. Thus either (13.26) is an identity in $t$ or it has less than $k$ solutions $t$. The minimality of the pair $(p, q)$ in (13.4) implies that at most one of the families (13.25) may give an identity in (13.26). As $v \leq 2 d^{2}$, we obtain (13.22).

14. Proof of Theorems 3 and 4 and of the corollaries. Let us begin with Theorem 4 . We proceed by induction on $r$. If $r=1$, the assertion follows from Proposition 12.2 and Lemma 13.7. Now suppose that $r>1$ and that the assertion holds true for $r-1$. The theorem then follows from Lemma 13.5(b), in fact (13.10) implies the bound (1.30).

Now let us turn to Theorem 3. If $r=1$, we have two possibilities. 
Either $f_{1}(x) \alpha_{1}^{x}=g_{1}(y) \beta_{1}^{y}$ is not exceptional. Then by Proposition 12.1 a set with $2^{s^{7} 2^{45 d !(2 k) !}}$ elements will suffice, plus additionally $2 d^{2} k$ elements from Lemma 13.8, and the theorem follows.

Or $f_{1}(x) \alpha_{1}^{x}=g_{1}(y) \beta_{1}^{y}$ is exceptional but not both $f_{0}$ and $g_{0}$ are constant. Then by Lemma 13.6 a set $M_{1}$ with $\leq 4 k^{2}$ elements suffices.

Now suppose that $r>1$. As $F, G$ is not exceptional, we may infer that there is a pair $i, j(0 \leq i<j \leq r)$ such that the system

$$
f_{i}(x) \alpha_{i}^{x}=g_{i}(y) \beta_{i}^{y}, \quad f_{j}(x) \alpha_{j}^{x}=g_{j}(y) \beta_{j}^{y}
$$

is not exceptional (in an obvious sense). So, for the set of solutions of (14.1) we may apply one of Lemmata 13.1-13.6, 13.8. The assertion follows at once as for the exceptional set of cardinality $\leq M_{1}$ the worst cases arise if one equation in (14.1) is not exceptional and the other involves roots of unity, or if both equations are as in Lemma 13.5. We may conclude that an exceptional set of cardinality

$$
\leq \max \left\{2^{2^{1080} s^{7}}, 2^{s^{7} 2^{45 d !(2 k) !}}+2 d^{2} k\right\}
$$

will suffice. The theorem follows.

As for Corollary 1, we clearly get the one-parameter family of solutions $m=t, n=t(t \in \mathbb{Z})$. If $\left\{u_{m}\right\}$ is symmetric, then we have relations (1.34). Hence $\alpha_{i} \alpha_{i+1}$ is a root of unity in $K$ and so by Lemma 6.1 we have $w \leq 2 d^{2}$, which gives the possible second family (1.39) by Theorem 3 . We still have to prove the bound (1.36).

We apply Theorems 2 and 3. By Theorem 2 the number of solutions (1.37) that do not satisfy $(1.20)$ or $(1.21 \mathrm{a}, \mathrm{b})$ has cardinality

$$
\leq 2^{s^{7} 2^{45 d !\left(2 k^{3}\right) !}} .
$$

From Theorem 3, applied to (1.20), and also to $(1.21 \mathrm{a}, \mathrm{b})$ if $\left\{u_{m}\right\}$ is symmetric, we get in addition a set of cardinality

$$
\leq 2 \max \left\{2^{2^{1080} s^{7}}, 2^{s^{7} 2^{46 d !(2 k) !}}\right\} .
$$

As we assume $k>1$, the sum of the bounds in (14.2), (14.3) does not exceed

$$
2^{s^{7} 2^{46 d !\left(2 k^{3}\right) !},}
$$

and Corollary 1 follows.

Finally, let us deal with Corollary 2. The linear family corresponding to equations (1.20), by Theorem 3 now has the shape $m(t)=t, n(t)=t+t_{0}$ $(t \in \mathbb{Z})$. It implies the polynomial identities

$$
f_{i}(t) \equiv \frac{b}{a} f_{i}\left(t+t_{0}\right) \alpha_{i}^{t_{0}} \quad(i=0, \ldots, r) .
$$


Hence we get $(b / a) \alpha_{1}^{t_{0}}=1$ and the identity $f_{1}(t) \equiv f_{1}\left(t+t_{0}\right)$. Now either $\operatorname{deg} f_{1}>0$ and $t_{0}=0$ or $\operatorname{deg} f_{1}=0$. In the latter case, as $k>1$, we have at least one further root $\alpha_{i}$ with $i \neq 1$ and $(b / a) \alpha_{i}^{t_{0}}=1$. But then by nondegeneracy $t_{0}=0$, and so in all cases $b / a=1$, which contradicts our hypothesis.

Now assume that $\left\{u_{m}\right\}$ is symmetric. As above in Corollary 1, we possibly get a one-parameter family $m(t)=w t+t^{\prime}, n(t)=-w t+t^{\prime \prime}(t \in \mathbb{Z})$ with $0<w \leq 2 d^{2}$, which induces the polynomial identities in $t$,

$$
\begin{aligned}
& f_{i}\left(w t+t^{\prime}\right) \alpha_{i}^{t^{\prime}}=\frac{b}{a} f_{i+1}\left(-w t+t^{\prime \prime}\right) \alpha_{i+1}^{t^{\prime \prime}} \\
& f_{i+1}\left(w t+t^{\prime}\right) \alpha_{i+1}^{t^{\prime}}=\frac{b}{a} f_{i}\left(-w t+t^{\prime \prime}\right) \alpha_{i}^{\prime \prime}
\end{aligned}
$$

Write $l=\operatorname{deg} f_{i}=\operatorname{deg} f_{i+1}$. Let $a_{i}$ and $a_{i+1}$ be the leading coefficients of $f_{i}$ and $f_{i+1}$ respectively.

Comparing the leading coefficients in (14.4) we get

$$
a_{i} w^{l} \alpha_{i}^{t^{\prime}}=(-1)^{l} \frac{b}{a} a_{i+1} w^{l} \alpha_{i+1}^{t^{\prime \prime}}, \quad a_{i+1} w^{l} \alpha_{i+1}^{t^{\prime}}=(-1)^{l} \frac{b}{a} a_{i} w^{l} \alpha_{i}^{t^{\prime \prime}},
$$

hence $\left(\alpha_{i} \alpha_{i+1}\right)^{t^{\prime}-t^{\prime \prime}}=(b / a)^{2}$. It follows that $b / a$ is a root of unity. The estimate (1.41) follows in the same way as (1.36) in Corollary 1.

\section{References}

[1] E. Dobrowolski, On a question of Lehmer and the number of irreducible factors of a polynomial, Acta Arith. 34 (1979), 391-401.

[2] J. H. Evertse, On sums of S-units and linear recurrences, Compositio Math. 53 (1984), 225-244.

[3] M. Laurent, Équations exponentielles polynômes et suites récurrentes linéaires, Astérisque 147-148 (1987), 121-139.

[4] -, Équations exponentielles-polynômes et suites récurrentes linéaires, II, J. Number Theory 31 (1989), 121-139.

[5] J. H. Loxton and A. J. van der Poorten, Multiplicative dependence in number fields, Acta Arith. 42 (1983), 291-302.

[6] G. Pólya und G. Szegö, Aufgaben und Lehrsätze aus der Analysis, II, 4. Auflage, Springer, Berlin, 1971.

[7] A. J. van der Poorten and H. P. Schlickewei, The growth condition for recurrence sequences, Macquarie Univ. Math. Rep. 82-00041, North Ryde, N.S.W., 1982.

[8] H. P. Schlickewei, S-unit equations over number fields, Invent. Math. 102 (1990), 95-107.

[9] —, Multiplicities of algebraic linear recurrences, Acta Math. 170 (1993), 151-180.

[10] H. P. Schlickewei and W. M. Schmidt, On polynomial-exponential equations, Math. Ann. 296 (1993), 339-361.

[11] - - - Linear equations in members of recurrence sequences, Ann. Scuola Norm. Sup. Pisa Cl. Sci. (4) 20 (1993), 219-246. 
[12] W. M. Schmidt, Diophantine Approximations and Diophantine Equations, Lecture Notes in Math. 1467, Springer, 1990.

ABTEILUNG MATHEMATIK II UNIVERSITÄT ULM

OBERER ESELSBERG

D-89069 ULM, GERMANY
DEPARTMENT OF MATHEMATICS UNIVERSITY OF COLORADO BOULDER, COLORADO 80309-0426 U.S.A. 